\title{
Topical Buparvaquone Nano-enabled Hydrogels for Cutaneous Leishmaniasis
}

Aikaterini Lalatsa $a^{a^{*}}$, Larry Statts ${ }^{a}$, Jéssica Adriana de Jesus ${ }^{b}$, Olivia Adewusia ${ }^{a}$, Maria Auxiliadora Dea-Ayuela ${ }^{c}$, Francisco Bolas-Fernandez ${ }^{d}$, Marcia Dalastra Laurentib, Luiz Felipe Domingues Passerob,e, Dolores R. Serranof.

${ }^{a}$ Biomaterials, Bio-engineering and Nanomedicines (BioN) laboratory, Institute of Biomedical and Biomolecular Sciences, School of Pharmacy and Biomedical Sciences, University of Portsmouth, White Swan Road, Portsmouth, PO1 2DT, UK.

bLaboratory of Pathology of Infectious Diseases (LIM-50), Medical School, University of São Paulo, Avenida Dr. Arnaldo 455, 01246903 Cerqueira César, SP, Brazil

'Departamento de Farmacia, Facultad de Ciencias de la Salud, Universidad CEU Cardenal Herrera, Edificio Seminario s/n, 46113-Moncada, Valencia, Spain

dDepartament of Microbiology and Parasitology, School of Pharmacy, Universidad Complutense de Madrid, Plaza Ramón y Cajal s/n, 28040, Madrid, Spain

eInstitute of Biosciences, São Paulo State University (UNESP), Praça Infante Dom Henrique s/n, 11330-900, São Vicente, SP, Brazil

IInstitute for Advanced Studies of Ocean Biosciences, São Paulo State University (UNESP), Av. João Francisco Bensdorp, 1178, 11350-011 São Vicente, SP, Brazil

9Departament of Pharmaceutics and Food Technology and Instituto Universitario de Farmacia Industrial (IUFI), School of Pharmacy, University Complutense de Madrid, Plaza Ramón y Cajal s/n, 28040, Madrid, Spain

\section{*Author for correspondence}

Dr. Aikaterini Lalatsa, Reader in Nanomedicines, School of Pharmacy and Biomedical Sciences, University of Portsmouth, White Swan Road, Portsmouth, PO1 2DT, UK, Email: katerina.lalatsa@port.ac.uk, Tel: +44 2392843929 


\section{Graphical Abstract}
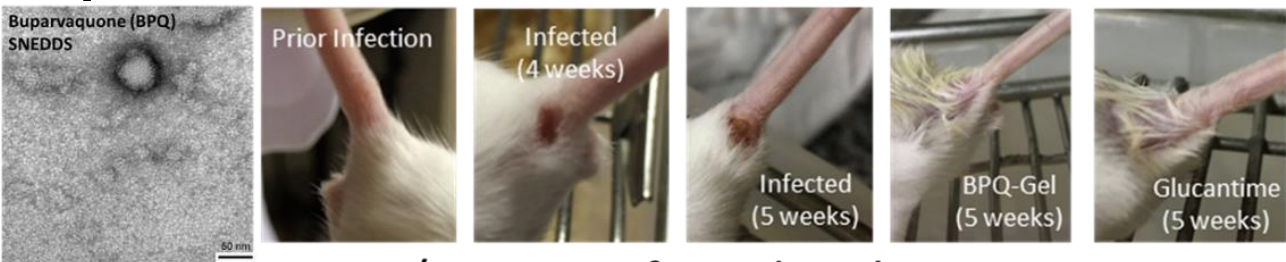

Buparvaquone (BPQ) SNEDDS Gel

BALB/c mice infected with L. Amazonensis
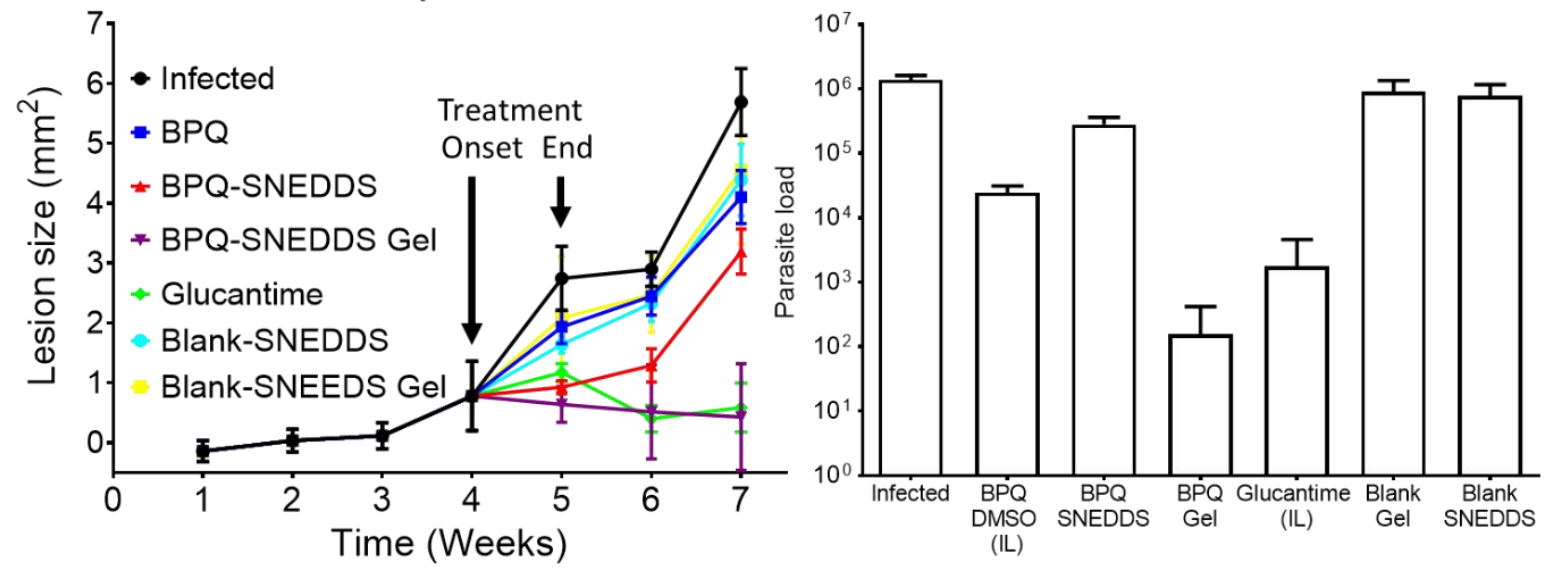


\section{Abstract}

Leishmaniasis is a neglected disease presenting cutaneous, mucosal and visceral forms and affecting an estimated 12 million mostly low-income people. Treatment of cutaneous leishmaniasis $(C L)$ is recommended to expedite healing, reduce risk of scarring, prevent parasite dissemination to other mucocutaneous (common with New World species) or visceral forms and reduce the chance of relapse, but remains an unmet need. Available treatments are painful, prolonged ( $>20$ days) and require hospitalisation, which increases the cost of therapy. Here we present the development of optimised topical self-nanoemulsifying drug delivery systems (SNEDDS) loaded with buparvaquone (BPQ, a hydroxynapthoquinone from the open Malaria Box) for the treatment of CL from New World species. The administration of topical BPQ-SNEDDS gels for 7 days resulted in a reduction of parasite load of $99.989 \pm 0.019 \%$ similar to the decrease achieved with intralesionally administered Glucantime $\AA(99.873 \pm 0.204 \%)$ in a L. amazonensis BALB/c model. In vivo efficacy was supported by ex vivo permeability and in vivo tape stripping studies. BPQSNEDDS and their hydrogels demonstrated linear flux across non-infected CD-1 mouse skin ex vivo of $182.4 \pm 63.0 \mu \mathrm{g} \mathrm{cm}^{-2} \mathrm{~h}^{-1}$ and $57.6 \pm 10.8 \mu \mathrm{g} \mathrm{cm}^{-2} \mathrm{~h}^{-1}$ respectively localising BPQ within the skin in clinically effective concentrations (227.0 $\pm 45.9 \mu \mathrm{g}$ and $103.8 \pm 33.8 \mu \mathrm{g}$ ) respectively. These levels are therapeutic as BPQSNEDDS and their gels showed nanomolar in vitro efficacy against $L$. amazonensis and $L$. braziliensis amastigotes with excellent selectivity index toward parasites versus murine macrophages. In vivo tape stripping experiments indicated localisation of $\mathrm{BPQ}$ within the stratum corneum and dermis. Histology studies confirmed the reduction of parasitism and indicated healing in animals treated with BPQ-SNEDDS hydrogels. These results highlight the potential clinical capability of nano-enabled $\mathrm{BPQ}$ hydrogels towards a non-invasive treatment for $\mathrm{CL}$.

\section{Key Words}

Buparvaquone, Cutaneous Leishmaniasis, Mucocutaneous Leishmaniasis, Selfnanoemulsifying drug delivery systems (SNEDDS), hydrogels, franz cell diffusion assays, tape stripping. 


\subsection{Introduction}

Leishmaniasis, a collection of parasitic diseases presenting cutaneous, mucosal and visceral forms caused by protozoa of the genus Leishmania spread in humans through the bite of a phlebotomine sandfly. Leishmaniasis remains amongst the most important parasitic diseases in the developing world [1], affecting an estimated 12 million mostly low-income people, while 1.3 million new cases per year are reported putting at risk over 310 million people [2]. Brazil alone accounts for a third of tegumentary cases reported in the Americas region [2]. Tegumentary leishmaniasis is divided into three major clinical phenotypes: localised cutaneous leishmaniasis $(\mathrm{CL})$, diffuse $\mathrm{CL}(\mathrm{DCL})$ and mucocutaneous leishmaniasis (MCL), whose manifestations range between small cutaneous nodules to gross mucosal tissue destruction. The clinical diversity is a consequence of the different Leishmania spp. responsible for the disease with New World species being more likely to result in clinical manifestations and complications such as MCL, the patient's immune response and the host susceptibility to the infection [3]. DCL and MCL forms cause severe disfigurement and disability resulting in gender inequality, stigmatisation and reduction of economic productivity, while the visceral (VL) form is fatal if left untreated (with a $100 \%$ fatality rate within 2 years) $[3,4]$.

Treatment of $\mathrm{CL}$ (even forms that can self-heal within 6-12 months) is recommended to expedite healing, reduce risk of scarring, prevent parasite dissemination to other forms (MCL or visceral leishmaniasis), and reduce the chance of relapse. A regime is decided based on clinical examination of the lesion, aetiological species and likelihood to progress to MCL. As there are no available vaccines, chemotherapy remains the mainstay of treatment [3]. Topical treatment is currently limited to the least severe forms of $\mathrm{CL}$ without the risk of dissemination and development of MCL. 
Available therapies are invasive [antimonials $\left(20 \mathrm{mg} \mathrm{Sb}^{\vee} \mathrm{kg}^{-1}\right.$ parenterally over 20 days), amphotericin B [5]] or poorly effective and subject to resistance (paromomycin, miltefosine), while possessing unacceptable toxicities [3]. Other local therapy approaches have also included physical methods (cryotherapy, thermotherapy, surgical removal and electrotherapy) [3]. However, the need for novel natural or synthetic molecules or repurposed drugs targeted via effective and safe delivery systems remains [6].

Buparvaquone (BPQ), a hydroxynapthoquinone with in vitro activity in the nanomolar range against Leishmania sp., failed to clinically translate as a viable treatment for VL due to its poor oral bioavailability limited by its poor aqueous solubility [3]. While the mechanism of action of BPQ is still unknown, it has been postulated to interfere with energy generation by inhibiting complex III (bc1 complex), the third complex in the electron transport chain, while being specific to non-host mitochondria [7]. BPQ, however, have favourable physicochemical characteristics for skin penetration such as low molecular weight, high $\log \mathrm{P}$ (5.63 [8] and 7.02 at $\mathrm{pH} 3.0$ [9]), a pka that falls within the range of average normal skin $\mathrm{pH}$, and relatively low melting point $\left(183.6^{\circ}\right)$ [1]. Due its high lipophilicity, BPQ can stay associated with cell membranes and not pass into aqueous layers or deeper skin layers (dermis), where a higher water content is present and where the leishmanial parasite reside $[9,10]$. No licensed $\mathrm{BPQ}$ formulations are currently available.

Few studies have tried to assess the ability of BPQ to permeate the stratum corneum (SC) and its efficacy topically in the treatment of $C L[10,11]$. Both a hydrous gel $(0.2 \%)$ and water-in-oil emulsion ( $1 \%$ cream) of buparvaquone significantly reduced cutaneous parasite burden ( $P<0.05,22$ days post-infection) and lesion size, compared with the untreated control $(P<0.0001,16$ days post-infection) [9]. Efficacy 
was better when BALB/c mice infected with L. major (non-cure model) were treated three days post-infection compared to 10 or 23 days post-infection [9]. However, studies in a self-cure model were not performed and levels of BPQ within the skin were not quantified.

We have recently reported the development of orally bioavailable BPQ selfnanoemulsifying drug delivery systems (SNEDDS) and solid SNEDDS for the treatment of VL with ability to elicit bioavailable BPQ levels and able to reduce parasite load in the spleen and to an extent in the liver [1]. Here we present the development of optimised topical SNEDDS $[12,13]$ with high BPQ loading and their respective hydrogels for the treatment of CL from New World species. Apart from permeability studies ex vivo using mouse skin, we have studied their in vivo efficacy and pharmacokinetics using tape stripping.

\subsection{Methods}

\subsection{Materials}

Buparvaquone (>95\%, HPLC) was obtained from Toronto Research Chemicals (Toronto, Canada). Labrasol (Caprylocaproyl macrogol-8 glycerides), Transcutol P (diethylene glycol monoethyl ether) and Capryol 90 (Propylene glycol monocaprylate) were donated from Gatefosse (Alpha Chemicals, Berkshire, UK). Carbomer (Carbopol 940) was purchased from Fisher Scientific UK (Loughborough, UK). Franz cells were specially made by Soham Scientific Ltd (Fordham, UK). STRAT-M artificial skin membranes $(25 \mathrm{~mm})$ were obtained from Millipore (Batch: K5JA1303, Watford, UK) and used according to manufacturer's recommendations. Cell culture media were bought from Sigma-Aldrich (Madrid, Spain).

\subsection{Excised mouse skin samples}


Male BALB/c (8 weeks, 25-28g, Harlan Iberica (Barcelona, Spain)) were euthanised, and the skin was removed. Skin samples were shaved using a scalpel and skin was placed in $60^{\circ} \mathrm{C}$ de-ionised water for 30 seconds, after which the underlying muscle tissue and hypodermis were manually removed. Skin was dried and placed flat in foil and freezed in water-impermeable plastic bags at $-50^{\circ} \mathrm{C}$ until required. Skin was used within a month of preparation [12] . When, thawed the epidermis was placed in phosphate buffer (1X, pH 7.4 $\pm 0.1,137 \mathrm{mM} \mathrm{NaCl}, 10 \mathrm{mM}$ Phosphate, $2.7 \mathrm{mM} \mathrm{KCl}$, $37^{\circ} \mathrm{C}$ ) prior being mounted on the Franz cells for diffusion assays.

\subsection{Preparation of buparvaquone SNEDDS (BPQ-SNEDDS)}

BPQ-SNEDDS (2 \% w/w) were prepared by dispersing BPQ (0.02g) within an isotropic mixture of Labrasol $(0.3 \mathrm{~g})$, Capryol $90(0.1 \mathrm{~g})$ and Transcutol P $(0.6 \mathrm{~g})$ respectively $[12,13]$. The ratio of oil:surfactant and solvent was optimised in terms of particle size using tertiary diagrams and choice of surfactants and solvents was based on solubility studies $[1,12,13]$. BPQ and Labrasol were vortexed followed by bath sonicating (150 Watts, P Selecta Ultrasons-H, Barcelona, Spain) for 15 minutes. When BPQ was completely dissolved, Capryol 90 and Transcutol P were added and left overnight in a water bath at $37^{\circ} \mathrm{C}$ (Memmert WNE 45, Schwabach, Germany) for at least 16 hours [1]. Blank SNEDDS were produced using this method but without adding BPQ.

\subsection{Preparation of BPQ-SNEDDS Gels $(1 \% \mathrm{w} / \mathrm{w})$}

Carbopol 940 (1 g) was added in de-ionised water $(25 \mathrm{~mL})$ and left to swell overnight. The $\mathrm{pH}$ of the swollen gel was then adjusted to $\mathrm{pH} 6.5$ by addition of sodium hydroxide $(\sim 0.25 \mathrm{ml}, 1 \mathrm{M})$. Neutralised Carbopol $940 \mathrm{gel}(1.5 \mathrm{~g})$ was mixed with BPQ-SNEDDS $(2 \% \mathrm{w} / \mathrm{w}, 1.5 \mathrm{~g})$ yielding a final hydrogel with a $\mathrm{pH}$ of $6.5 \pm 0.1$, (Accumet AB200 pH meter, Fisher Scientific, Loughborough, UK). 


\subsection{Measurement of particle size and zeta potential of prepared SNEDDS and}

SNEDDS loaded gels.

BPQ-SNEDDS and BPQ-SNEDDS gels were diluted 1 in 1000 w/w and 1 in 500 w/w with de-ionised water $(\mathrm{pH} 6.5 \pm 0.1)$. Diluted gels were centrifuged $(4,000 \mathrm{rpm} \approx$ 4,027 RCF, 5 minutes, SciSpin Micro Centrifuge, Shropshire, UK) to remove carbomer, and the particle size and zeta-potential was measured as previously described using a Nano-ZS Zetasizer (Malvern Instruments, Worcestershire, UK) [13-15].

\subsection{Morphology of BPQ-SNEDDS gels (TEM).}

A drop of the aqueous diluted BPQ-SNEDDS gels ( 1 in $2000 \mathrm{w} / \mathrm{w}$ ) was placed on a Formvar/Carbon coated grid (F196/100 3.05 mm, mesh 300, TAAB Labs Ltd, Berks, UK) prior staining with $1 \%$ uranyl acetate and transmission electron microscopy (TEM) imaging using a Joel JEM 1400 TEM microscope (Welwyn Garden City, UK) was used to image the particles as previously described [13-15]. Digital images were processed using an AMT (digital) camera.

\subsection{In vitro leishmanicidal activity and cytotoxicity studies}

L. amazonensis (MHOM/Br/79/Maria) and L. (V.) braziliensis (MHOM/Br/75/M2903) promastigotes, which were kindly provided by Prof. Alfredo Toraño (Carlos III Health Institute, Madrid, Spain), were used. Promastigotes were cultured in Schneider's Insect Medium (Sigma, St. Louis, MO, USA) at $26^{\circ} \mathrm{C}$ supplemented with $20 \%$ heatinactivated foetal bovine serum (FBS) (Sigma, St. Louis, MO, USA), penicillin (100 U $\left.\mathrm{mL}^{-1}\right)$ and streptomycin $\left(100 \mu \mathrm{gL}^{-1}\right)$ in culture flasks $(25 \mathrm{~mL})$. Promastigotes and intracellular susceptibility assay were conducted as previously described and summarised in SI [16, 17]. Mouse macrophage cytotoxicity assay were also performed as previously described and summarised in SI [1, 18, 19]. 


\subsection{In vitro Franz cells diffusion studies}

Modified Franz diffusion cells displayed an approximate diffusional area of $1.334 \pm$ $0.008 \mathrm{~cm}^{2}$ [12]. After the compartments were rinsed with phosphate buffer $(1 \mathrm{X}, \mathrm{pH}$ $7.4 \pm 0.1)$ and a $5 \mathrm{~mm}$ stirrer bar was added to the receiver compartment, the compartment was filled up with $2 \%$ hydroxyl propyl $\beta$ cyclodextrin (HP- $\beta-C y D$ ) in PBS which have been shown to be able to maintain sink conditions for BPQ [10]. STRAT-M or mouse skin with the stratum corneum facing upwards was clamped into the donor compartment. The two pieces were tightly sealed using Parafilm ${ }^{\mathrm{TM}}$ and clamped together. PBS $(1 \mathrm{~mL})$ was added onto the donor compartment and franz cells were placed into a water bath $\left(37^{\circ} \mathrm{C}, 250 \mathrm{rpm}, \mathrm{RCT}\right.$ basic, IKA ${ }^{\circledR}$ England Ltd, Oxford, UK). After 30 minutes, the receptor compartment buffer was removed for analysis and re-filled with fresh buffer pre-warmed at $37^{\circ} \mathrm{C}(2 \%$ HP- $\beta$-CyD PBS for BPQ) using a 21 gauze needle and $1 \mathrm{~mL}$ syringe. The PBS were discarded from the donor compartment and an excess of formulation (0.6 g) was added ensuring complete contact with the membrane or skin. At specific time intervals, samples (0.5

$\mathrm{mL}$ ) were collected from the receptor site using a syringe $(1 \mathrm{~mL})$ attached to a needle (21 gauge, $40 \mathrm{~mm}$ length). The samples were placed in HPLC vials ready for analysis. The receptor chamber was immediately refilled with pre-warmed buffer and the sampling port capped. All skin used was thawed from frozen on the day of study. After 6 hours of exposure, skin was wiped with an ethanol impregnated cotton bud and cut in half. The one half was fixed using formalin (10\%, neutral) for a minimum of 48 hours in the fridge till processed for histopathology studies as described below. The other half was left in the freezer $\left(-20^{\circ} \mathrm{C}\right)$ overnight.

The other half was homogenised with receptor compartment buffer $(1.5 \mathrm{~mL}, 2 \% \mathrm{HP}$ $\beta$-CyD PBS $(\sim 10 \mathrm{~mL} / \mathrm{g})$ to which a further $1.5 \mathrm{~mL}$ of methanol was added prior to 
being placed in the freezer $\left(-20^{\circ} \mathrm{C}\right)$ for 1 hour. Each sample was then vortexed for 5 minutes and then centrifuged $(5,000 \mathrm{rpm} \approx 5,033 \mathrm{RCF}$, for 10 minutes $)$. The supernatant was collected and the extraction with methanol $(1.5 \mathrm{~mL})$ was repeated another two times, pooling together all the supernatants after centrifugation. The supernatant was then analysed by HPLC as below.

All diffusion assay samples and extracted skin samples were analysed using a modified method based on a reverse phase HPLC previously validated $[1,20]$. The mobile phase consisted of $1 \%$ acetic acid $(\mathrm{AcOH})$ in water, acetonitrile $(\mathrm{ACN})$ and methanol [30:60:10 (v/v)]. The HPLC comprised from a Jasco DG-2080-53 Degasser attached to a PU-1580 Pump, an AS-2050 autosampler and aUV1575 UV/Vis detector se at $251 \mathrm{~nm}$. Standards and samples were eluted at $1.2 \mathrm{~mL} \mathrm{~min}^{-1}$ using a Water XTerra MS C18 column $(150 \times 4.6 \mathrm{~mm}, 3.5 \mu \mathrm{m})$ and the injection volume was set at $40 \mu \mathrm{L}$. A linear calibration curve between 0.0488 to $50 \mu \mathrm{g} \mathrm{mL}^{-1}$ was produced with a retention time of 16.13 minutes for $B P Q$.

\subsection{In vivo pharmacokinetic and tape stripping experiments}

NMRI male mice (8 weeks old, $25-28 \mathrm{~g})$ were randomly split into groups $(\mathrm{n}=3)$ and allowed food and water ad libitum. BPQ-SNEDDS $(250 \mathrm{mg}, 2 \% \mathrm{w} / \mathrm{w})$ or BPQSNEDDS-GEL (500 mg, 1\% w/w) were weighted and placed into a small plastic bag. The centre of the round plastic lids of tubes $\left(1 \mathrm{~cm}^{2}\right)$ were cut and the resulted ring or an $1.5 \mathrm{~mL}$ centrifuge tube (with the tip cut) were super glued (Loctite super glue-3 original) onto the mouse's back (middle upper area) [21], which was previously shaved with a razor gently, without causing any visual damage or pain. The tip of the plastic bag containing the formulations was cut and the gel was gently applied onto the centre of the lid, so as the formulations was in contact with the skin. In the case of BPQ-SNEDDS only, the tube was sealed with the cut tip using superglue to avoid 
formulation spillage and ingestion of the formulation by the animal. For gels, due to the site of application, animal was not able to ingest the formulation. A third group was utilised to assess the effect of pre-application of metallic microneedles prior application of the BPQ-SNEDDS as described above. A dermal stamp electric pen (Electric self-adjustable microneedle system: Derma pen, China) was used with a needle length of $0.4 \mathrm{~mm}$ at the lowest speed. Microneedles were applied on the shaved skin for one second and immediately after the liquid SNEDDS were applied on top. Mice were sacrificed at different time points $(0.5,1,2$ and 4 hours) and the skin was collected from the mice and stored in the $-20^{\circ} \mathrm{C}$ until further analysis.

Quantification of BPQ in skin was performed using the tape stripping technique [12, 22, 23]. The skin exposed to formulations were wiped with cotton buds and then with ethanol impregnated cotton buds. The distribution of BPQ across the stratum corneum and epidermis was determined by thirty sequential tape-strips $(10 \times 10 \mathrm{~mm}$, square tapes, double sided, Punts ${ }^{\circledR}$, Etyprinter S.L., Barcelona, Spain). Tapes were pressed for 5 seconds under slight manual pressure using a custom-made weight (15 mm $\varnothing, 100 \mathrm{~g}$ ). BPQ was extracted from each tape strip with acetonitrile: water (1:1, 0.5mL). Tapes were bath sonicated (Ultrasons-H Selecta, Madrid, Spain) for 10 minutes and centrifuged (5,000 $\times 5$ minutes). The supernatant was analysed by HPLC as above.

After the sequential 30 strips were removed, the area of the skin where the formulation was applied was cut out using scissors and skin was weighted and homogenised using a pestle and mortar using $2 \%$ HP- $\beta$-CyD PBS (10 $\mathrm{mL} \mathrm{g}^{-1}$ of skin). After homogenisation, an equal volume of methanol was added and the samples were centrifuged $(8,000 \mathrm{rpm} \approx 8,053 \mathrm{RCF}, 10$ minutes). The supernatant was analysed by HPLC as above. 


\subsection{Histopathological analysis of human and mouse treated skin.}

Fixed treated mouse skin $(6$ h) with BPQ-SNEDDS and BPQ-SNEDDS gels were prepared, stained (haematoxylin and eosin) and imaged as previously described [12, 24].

\subsection{Efficacy studies in L. (L.) amazonensis infected BALB/c mice.}

L. (L.) amazonensis parasite (MHOM/BR/73/M2269) was kindly provided by Prof. Dr. Fernando T. Silveira from the cryobank of "Leishmaniasis Laboratory Prof. Dr. Ralph Laison", Department of Parasitology, Evandro Chagas Institute, Ministry of Health, (Belém, Pará, Brazil). The parasite was identified using monoclonal antibodies and isoenzyme electrophoretic profiles at the Leishmaniasis Laboratory of the Evandro Chagas Institute. This parasite was grown in RPMI 1640 media (Gibco®, Life Technologies, Carlsbad, CA, USA), supplemented with $10 \%$ heat-inactivated fetal bovine serum, $50 \mu \mathrm{g} \mathrm{mL}^{-1}$ of gentamicin, and $50,000 \mathrm{IU} \mathrm{mL}^{-1}$ of penicillin (R10) at $25^{\circ} \mathrm{C}$. Promastigote forms in the stationary phase were used.

BALB/c mice (female, 6-8 weeks old) were obtained from Medical School of São Paulo University and divided in four groups of four animals per group. This study was carried out in strict accordance with the recommendations in the Guide for the Care and Use of Laboratory Animals of the Brazilian National Council of Animal Experimentation (http://www.cobea.org.br). The protocol was approved by the Committee on the Ethics of Animal Experiments of the Institutional Animal Care and Use Committee at the Medical School of São Paulo University (CEUA 322/12). For all experimental procedures, mice were anaesthetized with sodium thiopental ( $1 \mathrm{mg}$ $\left.200 \mu \mathrm{L}^{-1}\right)$. 
Animals were subcutaneously infected at the base of the tail with $1 \times 10^{6}$ promastigotes of $L$. (L.) amazonensis in stationary growth phase (final volume 25 $\mu L)$, except for the healthy control group which was given only physiological saline solution $(0.9 \% \mathrm{NaCl}, 25 \mu \mathrm{L})$. After 28 days of infection, treatment was started and animals were divided into the following groups: group 1 and 2 were topically treated with BPQ-SNEDDS gel (45 mg kg-1) and BPQ-SNEDDS (45 mg kg-1); group 3 and 4 were treated with intralesionally injected BPQ (in DMSO, $45 \mathrm{mg} \mathrm{kg}^{-1}$ ) and Glucantime (100 $\mathrm{mg} \mathrm{kg}^{-1}$ ) respectively; groups 5 and 6 were treated topically with blank SNEDDS gel and blank SNEDDS of equivalent weight respectively; group 7 involved the infected untreated animals and finally group 8 was the non-infected, untreated animals. Animals was treated with appropriate formulation once daily for a total of 7 days. Animal weight was monitored prior and 24 hours post infection and weekly thereafter. The development of lesions in infected and treated groups was measured weekly after infection. The size of the lesion was evaluated weekly by recording the average diameter of the tail measured as the mean of tail base diameters in horizontal and vertical directions using a caliper [25, 26]. Two weeks after the last treatment, animals were anaesthetized with sodium thiopental and euthanised by cardiac puncture. No animals needed to be euthanised prior the final endpoint of the study.

The parasite load was quantified using limiting dilution assays from skin from the point of parasite inoculation. Briefly, skin biopsies from different groups were aseptically excised and homogenized in Schneider's medium. The skin suspensions were subjected to 12 serial dilutions with four replicate wells. The number of viable parasites was determined based on the highest dilution that promastigotes could be grown after 10 days of incubation at $25^{\circ} \mathrm{C}$. The results were expressed as the mean 
\pm standard deviation of three independent experiments and the nonparametric Kruskal Wallis test was used to compare results among groups. Differences were considered statistically significant at $5 \%$ significance level $(p<0.05)$. GraphPad Prism 5 (GraphPad Software, Inc., La Jolla, CA, USA) was used to analyze the results. Skin biopsies from the inoculation site from treated and non-treated groups were also collected and fixed in buffered $5 \%$ formalin for histopathological studies as previously described [27].

\subsection{Results}

Prepared BPQ-SNEDDS and BPQ-SNEDDS gels illustrated sizes consistently below $400 \mathrm{~nm}$ (Table 1), good colloidal stability and spherical morphology (Figure 1). The particle size of BPQ SNEDDS and BPQ SNEDDS gels were similar indicating the ability of nanoparticles forming after dilution of the gels in aqueous environments [12]. High loading of BPQ $(2 \% \mathrm{w} / \mathrm{w})$ was possible in SNEDDS (higher than previous reports $[1,20])$ and able to elicit topical hydrogels with high loading (1\% w/w) [10], which would be clinically relevant if skin permeable [9]. Viscosity of the prepared hydrogels were appropriate for skin application avoiding running [12].

Table 1. Mean particle size, polydispersity and zeta potential of prepared batches of BPQ-SNEDDS and BPQ-SNEDDS gels $(n=4)$.

\begin{tabular}{|l|c|c|c|}
\hline Formulation & Particle size $(\mathrm{nm})$ & Polydispersity & Zeta Potential $(\mathrm{mV})$ \\
\hline Blank SNEDDS & $255 \pm 37$ & $0.685 \pm 0.085$ & $-13.5 \pm 0.2$ \\
\hline Blank SNEDDS gels & $294 \pm 11$ & $0.578 \pm 0.011$ & $-25.7 \pm 1.9$ \\
\hline BPQ-SNEDDS $(2 \%)$ & $260 \pm 35$ & $0.758 \pm 0.072$ & $-34.5 \pm 1.2$ \\
\hline BPQ-SNEDDS gel $(1 \%)$ & $266 \pm 99$ & $0.609 \pm 0.046$ & $-28.7 \pm 1.1$ \\
\hline
\end{tabular}




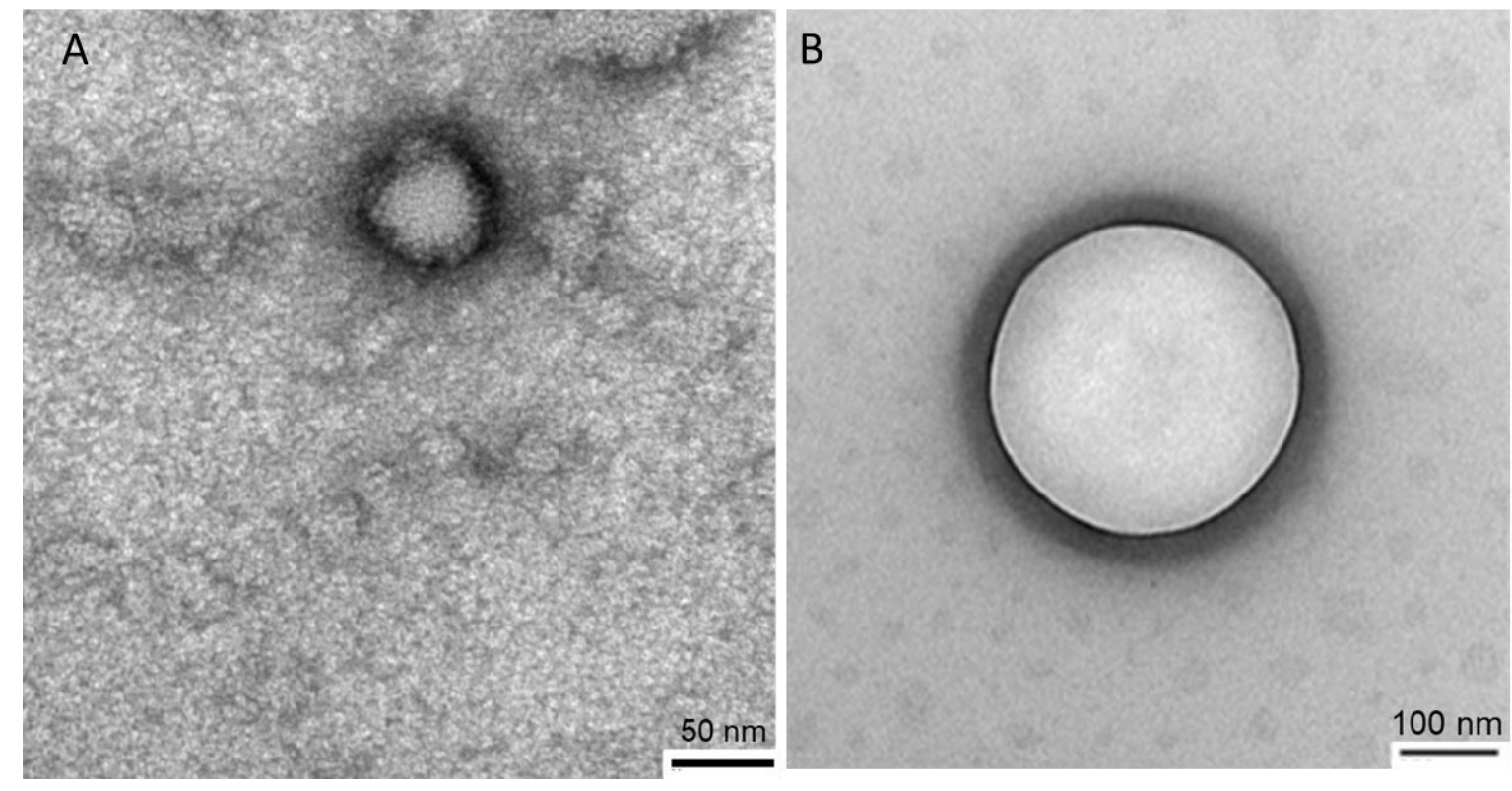

Figure 1. TEM images of aqueous dispersions of BPQ-SNEDDS (A) and BPQSNEDDS gel (B) (1 in $2000 \mathrm{w} / \mathrm{w}$ ) stained with 1\% uranyl acetate. Bars: 50 and 100 $\mathrm{nm}$ respectively.

Linear permeation profiles were observed for both BPQ-SNEDDS and BPQSNEDDS gels (Figure 2A, B). Across STRAT-M diffusion test models, BPQSNEDDS and their gels show short lag time $(4.2 \pm 4.2$ minutes and $18.84 \pm 3.96$ minutes respectively) and high flux $\left(30.7 \pm 2.6 \mu \mathrm{g} \mathrm{cm}^{-2} \mathrm{~h}^{-1}\right.$ and $12.0 \pm 1.3 \mu \mathrm{g} \mathrm{cm}^{-2} \mathrm{~h}^{-1}$ respectively) (Table 2, Figure 2A). BPQ flux across BALB/c full thickness skin was 3.7 fold for BPQ-SNEDDS and similar for BPQ-SNEDDS gel to previous published BPQ hydrous gels (BPQ-SNEDDS Jss: $1.726 \pm 0.650$, BPQ-SNEDDS gels Jss: $0.506 \pm 0.128 \mu \mathrm{g} \mathrm{cm}^{-2} \mathrm{~h}^{-1}$ and previously reported BPQ hydrous gels Jss: $0.47 \pm 0.03$ $\mu \mathrm{g} \mathrm{cm}{ }^{-2} \mathrm{~h}^{-1}$ [10]) (Table 2). High concentrations were detected within the homogenised skin and levels seem to be concentration driven with SNEDDS resulting in almost twice as high levels compared to gels (Table 2). Comparing the correlation co-efficient for various release mathematical models (Table 3), the Korsmeyer-Peppas model was found to best fit BPQ release from SNEDDS (anomalous non-Fickian), while a zero order release model better fits BPQ release 
from BPQ-SNEDDS gels indicating a time dependent and concentration independent mechanism (Table 3). The low lag time indicates that SNEDDS release from hydrogels is not hindered, possibly due to regions of low microviscosity of the carbomer as only $\sim 50 \%$ of the polymer chains are ionised at $\mathrm{pH} 6.5[12,28]$.

Table 2. Skin permeation parameters for BPQ across STRAT M and excised mouse skin from nano-enabled formulations.

\begin{tabular}{|c|c|c|}
\hline Permeation Parameters & BPQ-SNEDDS (2\%) & BPQ-SNEDDS gel (1\%) \\
\hline \multicolumn{3}{|c|}{ Strat-M membranes } \\
\hline Dose $(\mu \mathrm{g})$ & 12,000 & 6,000 \\
\hline JSS $0-180\left(\mu \mathrm{g} \mathrm{cm}^{-2} \mathrm{~h}^{-1}\right)$ & $30.68 \pm 2.56$ & $11.97 \pm 1.29$ \\
\hline Lag time $(\mathrm{h})$ & $0.07 \pm 0.07$ & $0.31 \pm 0.07$ \\
\hline$Q_{60}\left(\mu \mathrm{g} \mathrm{cm}^{-2}\right)$ & $33.26 \pm 9.84$ & $7.83 \pm 1.15$ \\
\hline$Q_{180}\left(\mu \mathrm{g} \mathrm{cm}^{-2}\right)$ & $88.56 \pm 9.71$ & $28.94 \pm 2.77$ \\
\hline $\mathrm{Kp}(\mathrm{cm} / \mathrm{h})$ & $2.5610^{-3} \pm 0.2210^{-3}$ & $2.0010^{-3} \pm 0.2210^{-3}$ \\
\hline $\mathrm{D}$ app $\left(\mathrm{cm}^{2} / \mathrm{h}\right)$ & $7.6710^{-5} \pm 0.6510^{-5}$ & $5.9710^{-5} \pm 0.6410^{-5}$ \\
\hline Thickness (cm) & 0.030 & 0.030 \\
\hline \multicolumn{3}{|c|}{ BALB/c mouse skin } \\
\hline Dose $(\mu \mathrm{g})$ & 12,000 & 6,000 \\
\hline JSS $0-360\left(\mu \mathrm{g} \mathrm{cm}^{-2} \mathrm{~h}^{-1}\right)$ & $1.73 \pm 0.65$ & $0.51 \pm 0.13$ \\
\hline Lag time $(\mathrm{h})$ & $0.45 \pm 0.19$ & ND \\
\hline$Q_{60}\left(\mu \mathrm{g} \mathrm{cm}^{-2}\right)$ & $1.15 \pm 0.83$ & $0.71 \pm 0.15$ \\
\hline$Q_{180}\left(\mu \mathrm{g} \mathrm{cm}^{-2}\right)$ & $4.09 \pm 2.01$ & $1.47 \pm 0.27$ \\
\hline $\mathrm{Kp}(\mathrm{cm} / \mathrm{h})$ & $1.4410^{-4} \pm 0.5410^{-4}$ & $8.4310^{-4} \pm 0.2110^{-4}$ \\
\hline D app $\left(\mathrm{cm}^{2} / \mathrm{h}\right)$ & $1.1010^{-5} \pm 0.4710^{-5}$ & $6.5110^{-6} \pm 1.5710^{-6}$ \\
\hline Thickness (cm) & $0.08 \pm 0.01$ & $0.08 \pm 0.00$ \\
\hline Homogenised skin $(\mu \mathrm{g} / \mathrm{g})$ & $613.7 \pm 196.8$ & $334.7 \pm 103.1$ \\
\hline Homogenised skin $\left(\mu \mathrm{g} / \mathrm{cm}^{2}\right)$ & $170.2 \pm 34.4$ & $77.8 \pm 25.3$ \\
\hline \multicolumn{3}{|c|}{$\begin{array}{l}\text { Key; Dapp: Apparent diffusion co-efficient, Jss } 0-180 \text { : Flux at steady state (0-180 } \\
\text { minutes), Jsso-360: Flux at steady state (0-360 minutes), Kp: Permeability co- } \\
\text { efficient, ND: Not detected, } Q_{60}, Q_{180} \text { : Cumulative amount permeated after } 60 \\
\text { or } 180 \text { minutes. }\end{array}$} \\
\hline
\end{tabular}

Table 3. BPQ release kinetics from BPQ-SNEDDS and BPQ-SNEDDS gels across $\mathrm{BALB} / \mathrm{c}$ mouse skin.

\begin{tabular}{|l|c|c|c|c|c|}
\hline Formulation & $\begin{array}{c}\text { Zero Order } \\
\left(\mathrm{R}^{2}\right)\end{array}$ & $\begin{array}{c}\text { First Order } \\
\left(\mathrm{R}^{2}\right)\end{array}$ & $\begin{array}{c}\text { Higuchi } \\
\left(\mathrm{R}^{2}\right)\end{array}$ & $\begin{array}{c}\text { Hixson - } \\
\text { Crowell }\left(\mathrm{R}^{2}\right)\end{array}$ & $\begin{array}{c}\text { Korsmeyer - } \\
\text { Peppas }\left(\mathrm{R}^{2}\right)\end{array}$ \\
\hline BPQ-SNEDDS & 0.986 & 0.986 & 0.848 & 0.873 & $\begin{array}{c}\mathbf{0 . 9 9 7} \\
(\mathrm{n}=1.400)\end{array}$ \\
\hline $\begin{array}{l}\text { BPQ-SNEDDS } \\
\text { gels }\end{array}$ & $\mathbf{0 . 9 8 7}$ & 0.987 & 0.907 & 0.858 & $\begin{array}{c}0.960 \\
(\mathrm{n}=0.691)\end{array}$ \\
\hline
\end{tabular}



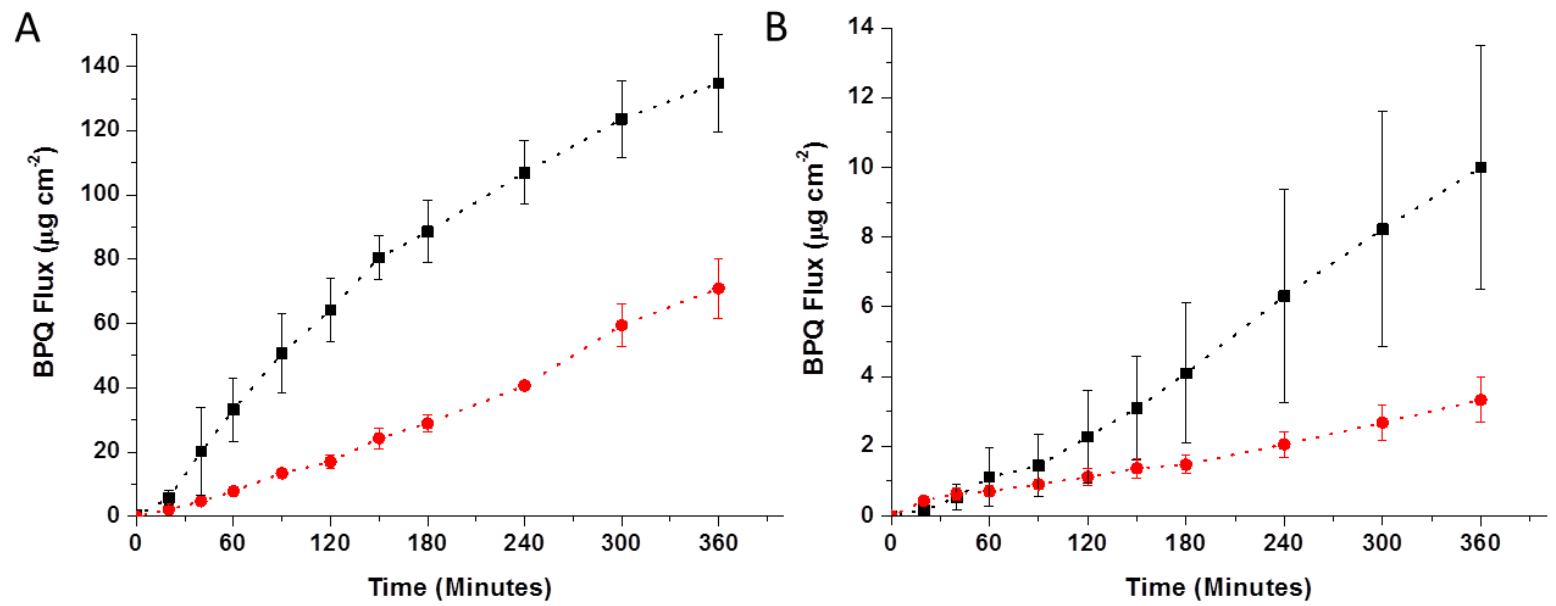

Figure 2. Flux of $B P Q$ across artificial skin/STRAT-M membraned $(A)$ and BALB/C excised mouse skin (B) [BPQ-SNEDDS (2\%, black squares), BPQ-SNEDDS gels $(1 \%$, red cycles $)]$.

In vivo tape stripping was undertaken to access $\mathrm{BPQ}$ levels within the SC and dermis after $0.5,1,2$, and 4 hours of exposure to the nano-enabled formulations immediately after the animals were euthanised. The data from the first three tapes were not taken into consideration as levels can be affected by left over formulation on the skin [9]. Considering that the thickness of the SC from the back of NMRI mice is approximately $15 \mu \mathrm{m}[9,29]$, the concentration in the SC (15 $\mu \mathrm{m} \sim 15$ tapes), dermis $(\sim 12 \mu \mathrm{m})$ and remaining homogenised skin is summarised in Table 4. Three of the selected time points for tape stripping experiments (i.e. 1, 2, and 4 hours) based on previously calculated lag times (Table 2) were collected after steady state was established (texp $>1.7$ tlag) [30], while at $0.5 \mathrm{~h}$ steady state was not achieved for BPQ-SNEDDS (but was achieved for the BPQ-SNEDDS gels). This is supported by levels obtained after tape stripping for BPQ-SNEDDS at $0.5 \mathrm{~h}$ compared to 1,2 , and $4 \mathrm{~h}$ respectively (Figure 3A, 3B, p $<0.05$ One-way ANOVA, Tukey's post-hoc test). As lag time for BPQ gels is well below 30 minutes, similar levels were obtained per tape when animals were exposed to BPQ-SNEDDS gels (Figure 3C, 3D, p>0.05 One-way ANOVA, Tukey's post-hoc test). However, an increased deviation in BPQ levels in 
tape strips obtained from animals exposed to the gels was observed. As animals used were not nude, presence of hair might limit the contact with the gel but not with the liquid SNEDDS and can potentially explain the enhanced observed variation in this group (Figure 3C, 3D, 4 Table 4). Application of microneedles (which can elicit pores that typically heal after 1 hour) demonstrate that permeation of SNEDDS is likely to be intracellular and intercellular as initial application seem to limit available surface area for permeation which limits levels in the dermis after 0.5 and $1 \mathrm{~h}$ exposure. However, in the long run when skin has healed (2 and 4 h), initial application of microneedles results in an enhanced permeation that increases dermis levels compared to SNEDDS alone (Table 4, Figure 3E, 3F, 4) $(p<0.05$, Student Ttest).

Table 4. Buparvaquone (BPQ) levels identified in stratum corneum, dermis and homogenised skin after in vivo exposure to the nano-enabled formulations.

\begin{tabular}{|c|c|c|c|c|}
\hline Formulation & $\begin{array}{l}\text { Expo- } \\
\text { sure }(h)\end{array}$ & $\mathrm{SC}(\mu \mathrm{g})$ & Dermis $(\mu \mathrm{g})$ & $\begin{array}{c}\text { Homogenised Skin } \\
(\mu \mathrm{g} / \mathrm{g})\end{array}$ \\
\hline \multirow{4}{*}{$\begin{array}{l}\text { BPQ-SNEDDS } \\
(2 \%)\end{array}$} & 0.5 & $32.923 \pm 0.923$ & $13.663 \pm 0.534$ & ND \\
\hline & 1 & $42.324 \pm 0.408$ & $18.722 \pm 0.537$ & $19.209 \pm 0.801$ \\
\hline & 2 & $44.172 \pm 0.063$ & $21.311 \pm 0.277$ & $11.877 \pm 12.061$ \\
\hline & 4 & $46.333 \pm 0.084$ & $23.183 \pm 0.027$ & $13.844 \pm 9.852$ \\
\hline \multirow{4}{*}{$\begin{array}{l}\text { BPQ-SNEDDS gel } \\
(1 \%)\end{array}$} & 0.5 & $22.465 \pm 10.529$ & $4.437 \pm 4.739$ & ND \\
\hline & 1 & $19.153 \pm 7.384$ & $1.306 \pm 0.943$ & $\mathrm{NQ}$ \\
\hline & 2 & $21.668 \pm 4.263$ & $3.441 \pm 3.470$ & $\overline{N Q}$ \\
\hline & 4 & $32.843 \pm 8.758$ & $6.587 \pm 5.739$ & $\mathrm{NQ}$ \\
\hline \multirow{4}{*}{$\begin{array}{l}\mathrm{MN}+\mathrm{BPQ} \\
\text { SNEDDS (2\%) }\end{array}$} & 0.5 & $35.581 \pm 8.196$ & $7.372 \pm 3.463$ & ND \\
\hline & 1 & $45.532 \pm 1.277$ & $15.869 \pm 5.280$ & $28.834 \pm 1.425$ \\
\hline & 2 & $47.870 \pm 0.015$ & $27.349 \pm 0.296$ & $4.979 \pm 2.824$ \\
\hline & 4 & $52.692 \pm 0.501$ & $27.815 \pm 1.597$ & $5.929 \pm 3.019$ \\
\hline
\end{tabular}



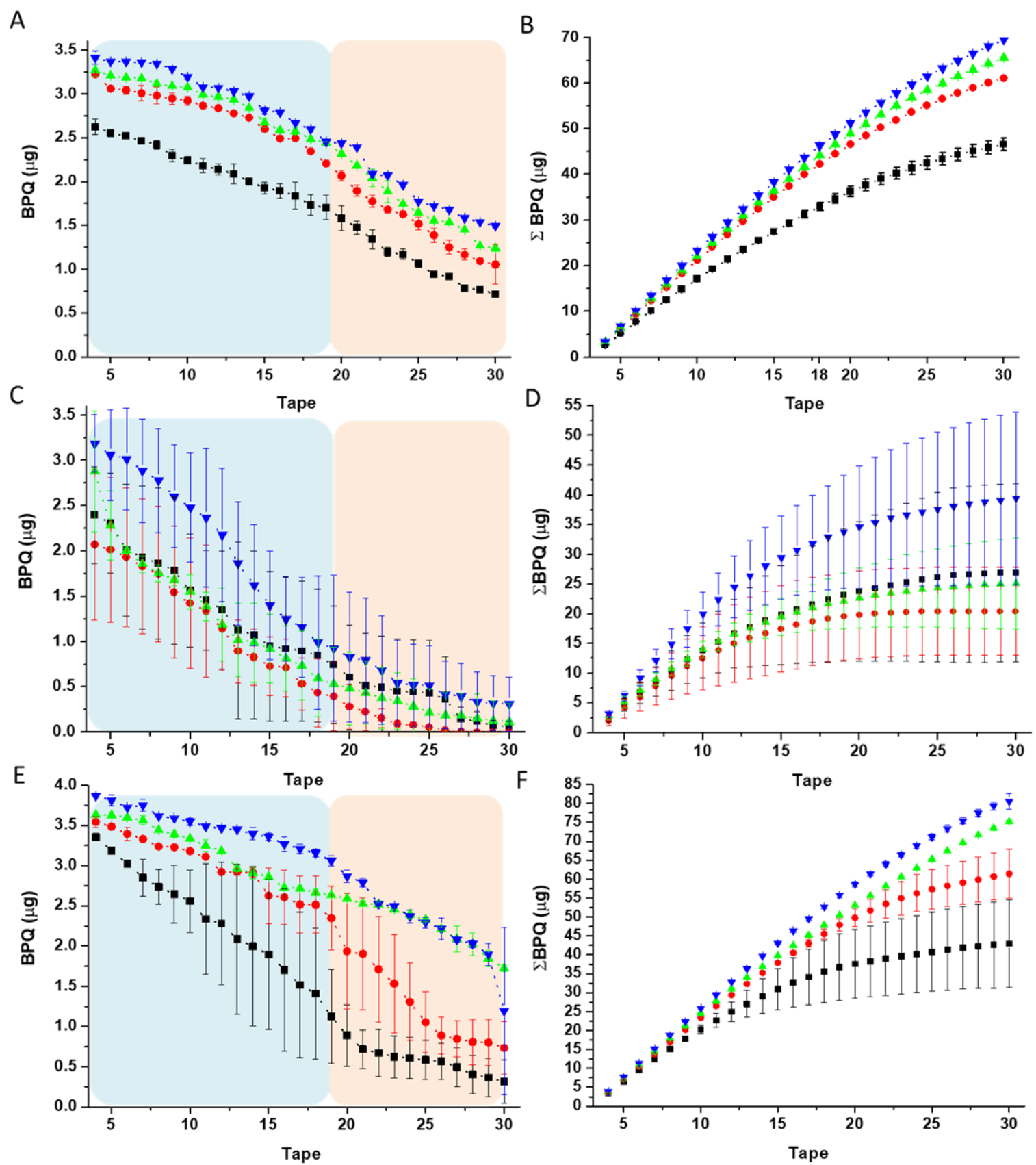

Figure 3. In vivo tape stripping after exposure to nano-enabled formulations; Amount of $B P Q$ per tape (1A) and cumulative BPQ amount (1B) after exposure to BPQSNEDDS over $0.5 \mathrm{~h}$ (black squares), $1 \mathrm{~h}$ (red cycles), $2 \mathrm{~h}$ (green triangles) and $4 \mathrm{~h}$ (blue inverted triangles). Amount of BPQ per tape (1C) and cumulative BPQ amount (1D) after exposure to BPQ-SNEDDS gels over $0.5 \mathrm{~h}$ (black squares), $1 \mathrm{~h}$ (red cycles), $2 \mathrm{~h}$ (green triangles) and $4 \mathrm{~h}$ (blue inverted triangles). Amount of BPQ per tape (1A) and cumulative BPQ amount (1B) after exposure to metallic microneedles $(0.4 \mathrm{~mm}, 1 \mathrm{sec})$ and BPQ-SNEDDS over $0.5 \mathrm{~h}$ (black squares), $1 \mathrm{~h}$ (red cycles), $2 \mathrm{~h}$ (green triangles) and $4 \mathrm{~h}$ (blue inverted triangles). 

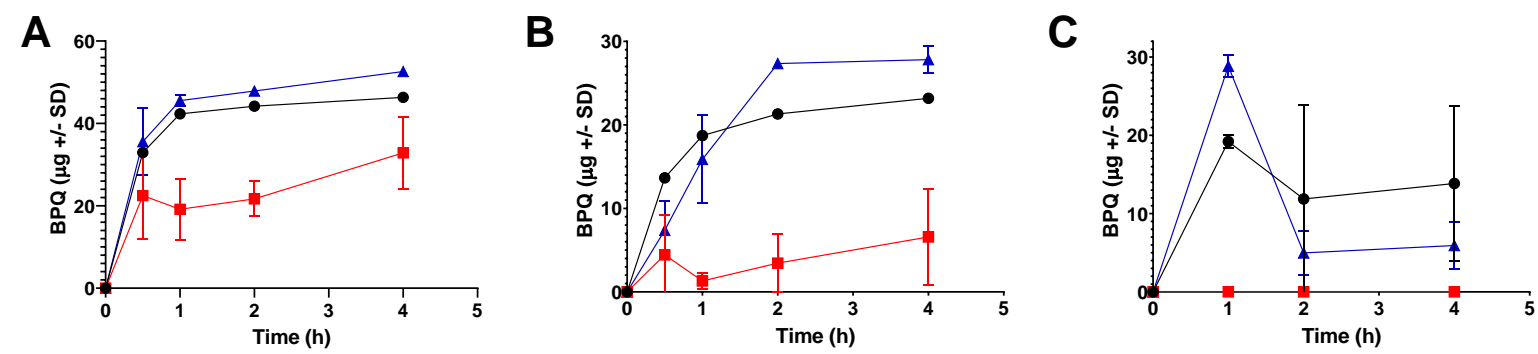

Figure 4. Amount of $B P Q$ recovered in $S C(A)$, dermis (B) and homogenised skin (C) over time after exposure to BPQ-SNEDDS (black circles), BPQ-SNEDDS gels (red squares), and metallic microneedles $(0.4 \mathrm{~mm}, 1 \mathrm{sec}$ ) and BPQ-SNEDDS (blue triangles) $(n=3)$. SD stands for standard deviation.

BPQ-SNEDDS and gels demonstrated nanomolar efficacy in $L$. amazonensis and $L$. amazonensis promastigotes and amastigotes forms (Table 5). BPQ nano-enabled topical formulations showed excellent selectivity for parasites compared to murine macrophages and a favourable target product profile for clinical translation [31].

Table 5. Parasiticidal activity and cytotoxicity of BPQ nano-enabled formulations in New World Leishmania sp.

\begin{tabular}{|c|c|c|c|c|c|}
\hline \multicolumn{5}{|c|}{ L. amazonensis } & \multirow{2}{*}{$\begin{array}{l}\text { J774 murine } \\
\text { macrophages } \\
\left(\mathrm{ng} \mathrm{mL}^{-1}\right)^{*}\end{array}$} \\
\hline Formulations & $\begin{array}{l}\text { Promastigotes } \\
\left(\mathrm{ng} \mathrm{mL}^{-1}\right)\end{array}$ & S.I. & $\begin{array}{l}\text { Amastigotes } \\
\text { (ng mL-1) }\end{array}$ & S.I. & \\
\hline Miltefosine & $12,440 \pm 490$ & 4.45 & $20,090 \pm 1470$ & 2.75 & $55,400 \pm 4,190$ \\
\hline $\mathrm{BPQ}$ (DMSO) & $0.32 \pm 0.01$ & 78125 & $37.30 \pm 3.20$ & 670 & $>25,000$ \\
\hline $\begin{array}{l}\text { BPQ- } \\
\text { SNEDDS }\end{array}$ & $0.63 \pm 0.07$ & 12519 & $79.30 \pm 10.00$ & 99.62 & $7,900 \pm 723.70$ \\
\hline $\begin{array}{l}\text { BPQ- } \\
\text { SNEDDS gels }\end{array}$ & $5.98 \pm 1.11$ & 645.6 & $2.67 \pm 0.58$ & 1452 & $3,861 \pm 735.20$ \\
\hline \multicolumn{5}{|c|}{ L. braziliensis } & \multirow{2}{*}{$\begin{array}{l}\text { J774 murine } \\
\text { macrophages } \\
\left(\mathrm{ng} \mathrm{mL}^{-1}\right)^{*}\end{array}$} \\
\hline Formulations & $\begin{array}{l}\text { Promastigotes } \\
\text { (ng mL-1) }\end{array}$ & S.I. & $\begin{array}{l}\text { Amastigotes } \\
\text { (ng mL-1) }\end{array}$ & S.I. & \\
\hline Miltefosine & $7280 \pm 350$ & 7.6 & $9950 \pm 1380$ & 5.56 & $55,400 \pm 4,190$ \\
\hline BPQ (DMSO) & $<1$ & $>25,000$ & ND & ND & $>25,000$ \\
\hline $\begin{array}{l}\text { BPQ- } \\
\text { SNEDDS }\end{array}$ & $0.79 \pm 0.06$ & 10,025 & $<1.525$ & $>5,180$ & $7,900 \pm 723.70$ \\
\hline $\begin{array}{l}\text { BPQ- } \\
\text { SNEDDS gels }\end{array}$ & $1.88 \pm 0.34$ & 2,053 & $31.67 \pm 12.00$ & 128.95 & $3,861 \pm 735.20$ \\
\hline
\end{tabular}




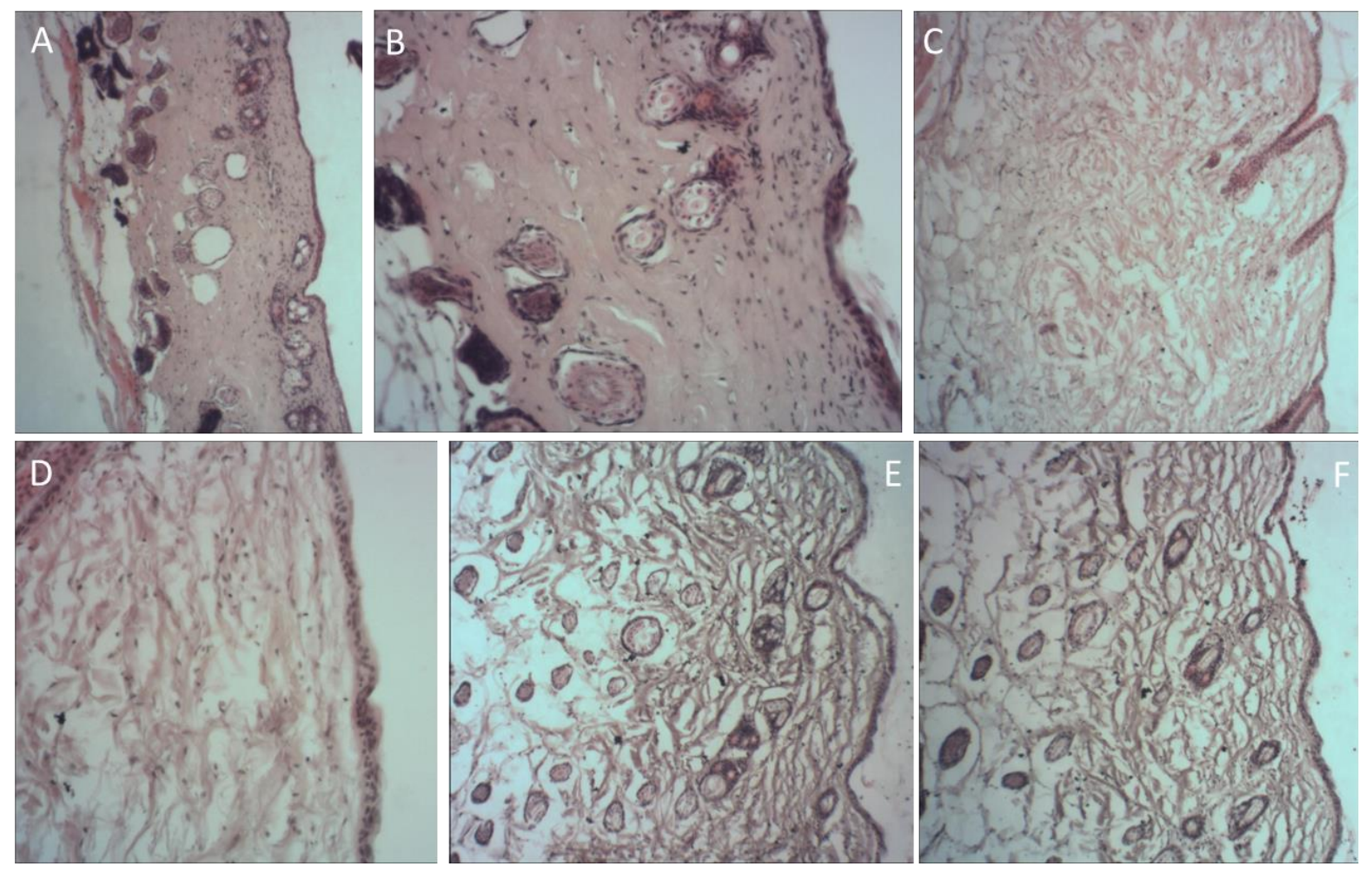

Figure 5. Micrographs of excised BALB/c mouse skin; Untreated skin (A: $x 4$ magnification and $B$ : $x 10$ magnification), skin exposed to BPQ-SNEDDS for 6 hours (C: $x 4$ magnification and D: $x 10$ magnification), and skin exposed to BPQ-SNEDDS gels for 6 hours (E: $x 4$ magnification and $F$ : $x 10$ magnification).
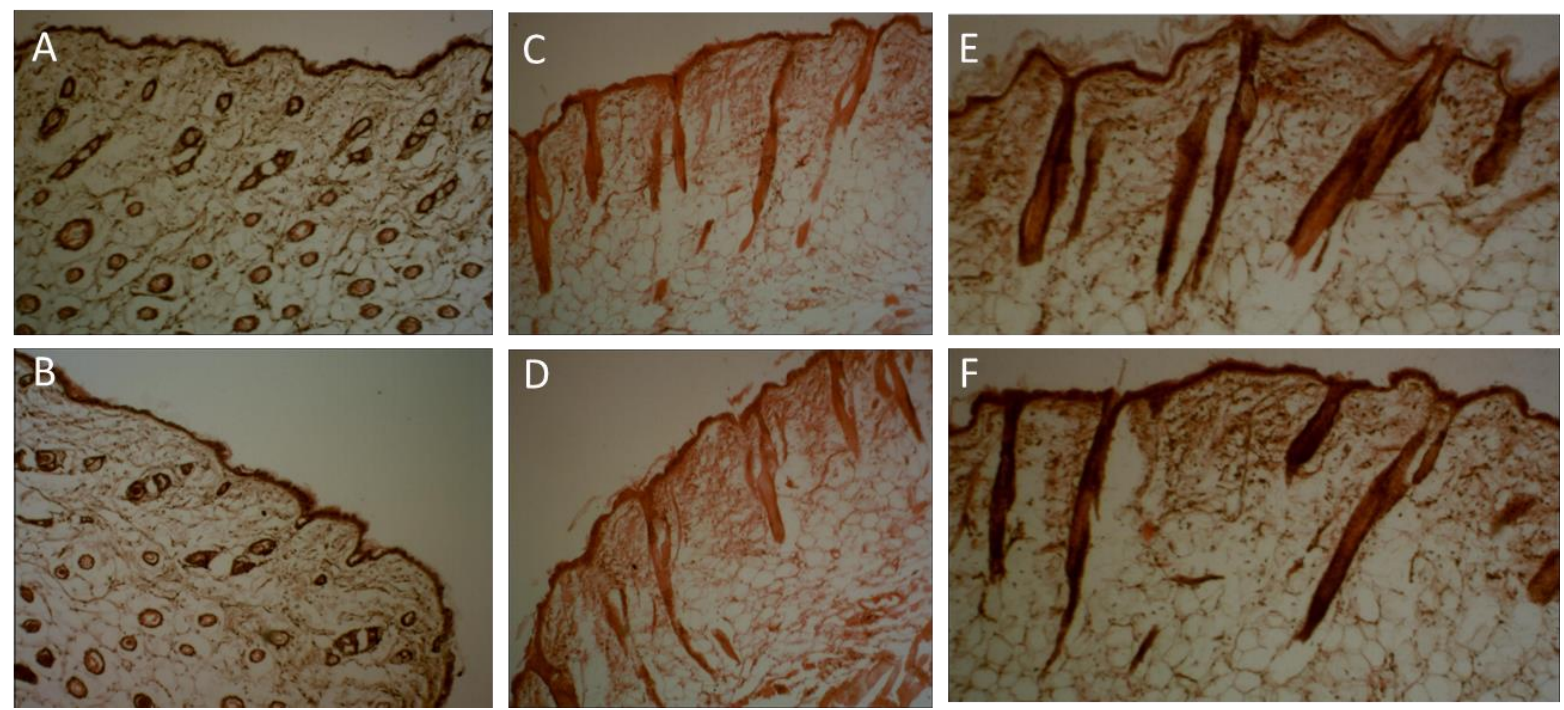

Figure 6. Micrographs of excised BALB/c mouse skin after in vivo administration of topical formulations; $B A L B / C$ skin exposed to BPQ-SNEDDS for 2 hours (A: $x 4$ magnification) and BPQ-SNEDDS gels for 2 hours (B: $x 4$ magnification), and BALB/C skin perforated by microneedles and exposed to BPQ-SNEDDS for 2 hours (C,D,E,F: x4 magnification). Healing of microneedle punctures is visible. 
Histopathology studies of BALB/c mouse skin demonstrated normal morphology with visualised hair follicles and sebaceous glands. After exposure to BPQ-SNEDDS (ex vivo and in vivo) and their gels, there were no signs of skin alterations (e.g. inflammation or erythema) following treatment with BPQ-SNEDDS, BPQ-SNEDDS gel (Figure 5 and Figure 6). Epidermal and dermal layers were devoid of inflammatory cells with absence of acanthosis or hyperkeratosis for both formulations.

The administration of topical BPQ-SNEDDS gels for 7 days resulted in a reduction of parasite load of $99.989 \pm 0.019 \%$ similar to the decrease achieved with intralesionally administered Glucantime ${ }^{\circledR}(99.873 \pm 0.204 \%)$ with a p-value $>0.05$ (Figure $7 \mathrm{C}$ ). When evaluating the lesion size, a decrease was observed for BPQSNEDDS gels and Glucantime ${ }^{\circledR}$ from the fifth week post-infection, while the untreated group showed an linear increase in lesion size over time. (Figure 7). Intralesional administration of BPQ in dimethylsulfoxide resulted in $98.241 \pm 0.491 \%$ reduction of parasite load, while BPQ-SNEDDS resulted in a $79.814 \pm 5.852 \%$. This is possibly explained as BPQ in dimethylsulfoxide is solubilised and when intralesionally injected is able to reach parasites at the dermis. The low level of moisture on skin surface, can hinder the BPQ-SNEDDS emulsification which can potentially result in unstable particles and even precipitation of BPQ. However, BPQSNEDDS managed to result in a reduction in lesion size (Figure 7B). 
A

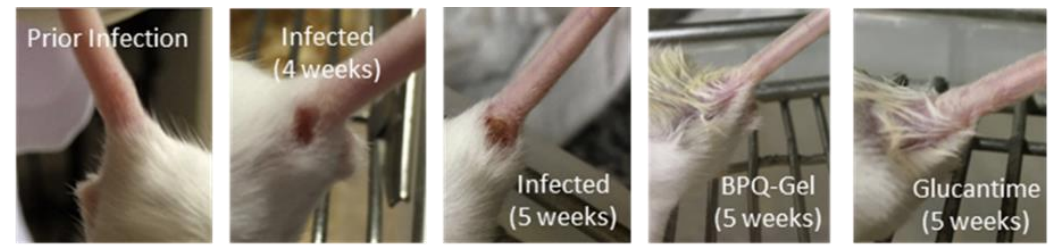

$\mathrm{BALB} / \mathrm{c}$ mice infected with $L$. Amazonensis
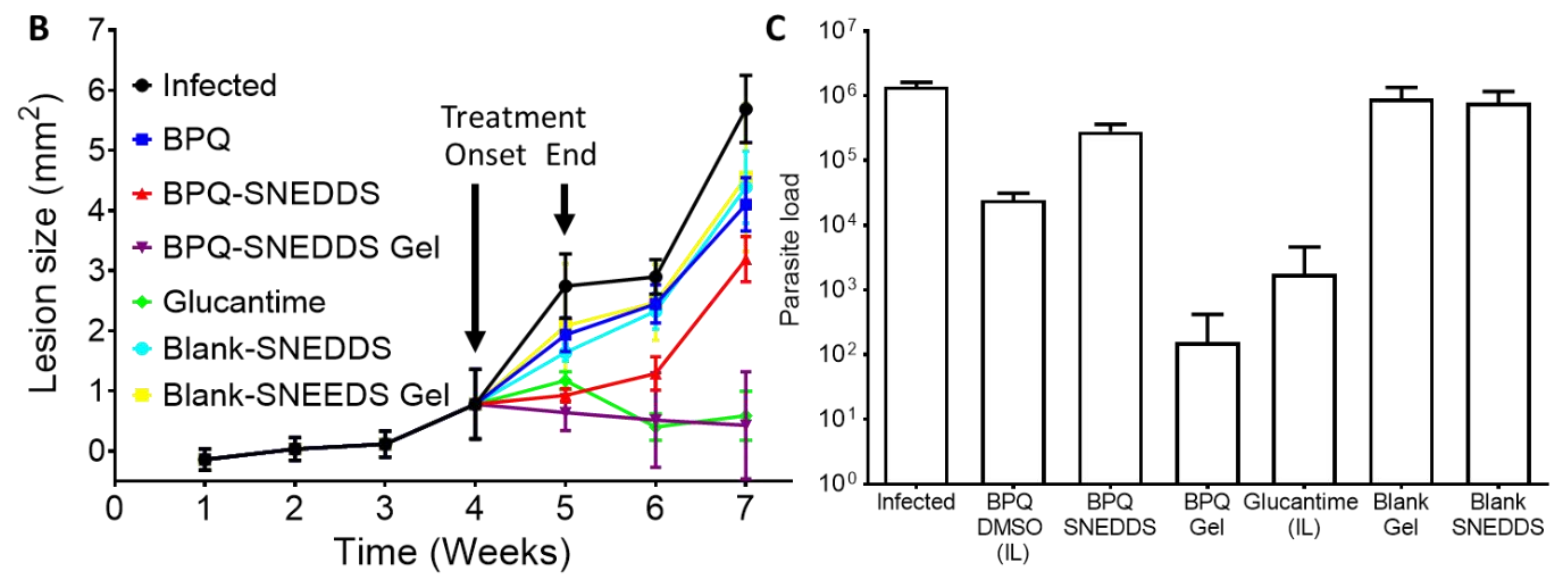

Figure 7. In vivo efficacy of nanoenabled gels in L. (L.) amazonensis infected BALB/C mice ( $n=8 /$ group). Macroscopic images of lesions of infected untreated and treated animals (A), lesion size (B) and parasite load (C) is shown for mice that were subcutaneously infected into the base of the tail with $10^{6}$ promastigotes of $L$. (L.) amazonensis. Four weeks after infection, mice were divided randomly into groups and treated once daily for 7 days; infected control (no treatment, black line), BPQ in DMSO intralesionally (IL) $\left(50 \mu \mathrm{L}, 45 \mathrm{mg} \mathrm{kg}^{-1}\right.$, blue line), BPQ-SNEDDS topically $\left(0.05 \mathrm{~g}, 45 \mathrm{mg} \mathrm{kg}^{-1}\right.$, red line), BPQ-SNEDDS gels topically $\left(0.15 \mathrm{~g}, 45 \mathrm{mg} \mathrm{kg}^{-1}\right.$, purple line), Glucantime IL(25 $\mu \mathrm{L}, 100 \mathrm{mg} \mathrm{kg}^{-1}$, green line), blank SNEDDS topically $(0.05 \mathrm{~g}$, turquoise line), blank SNEDDS gels topically $(0.15 \mathrm{~g}$, yellow line). No statistical differences in lesion size were obtained for blank SNEDDS, blank-SNEDDS gels and BPQ in DMSO at any time point (Kruskal Wallis test, 95\% level of significance). At week 5,6 , and 7 , there were statistical differences (reduction in lesion size) between the untreated group and groups that received Glucantime (week $5 p$ value:0.0282, week 6 p value: $<0.0001$, week 7 p value: $<0.0001)$, BPQ-SNEDDS gels topically (week 5 p value: $<0.0001$, week 6 p value: 0.0002 , week $7 p$ value: $<0.0001$ ) and BPQ-SNEDDS topically (week $5 p$ value:0.0002, week $6 p$ value: 0.0099 , week $7 p$ value: 0.0031) (Kruskal Wallis test, 95\% level of significance). BPQ in DMSO (IL), BPQ-SNEDDS topically, BPQ-SNEDDS gel topically, Glucantime (IL) have a significantly different parasite load compared to the untreated group $(p<0.0001$, OneWay Anova, Dunnett's test). Analysis was undertaken using Graphpad Prism 8.02 and results are shown as mean \pm SD.

The skin of infected untreated animals (Figure 8A) and infected animals treated with blank SNEDDS gels nanogel (Figure 8B) or blank SNEDDS (Figure 8C) showed intense skin parasitism and the inflammatory infiltrate was composed basically by 
mononuclear cells with areas of tissue necrosis (data not showed). Conversely, animals treated with $\mathrm{BPQ}$ in DMSO or BPQ-SNEDDS (Figure $8 \mathrm{D}$ and $8 \mathrm{~F}$ respectively) showed reduced parasitism compared to infected, untreated animals and although inflammatory process was present, it was less pronounced than in the untreated group. Animals treated with BPQ-SNEDDS gel (Figure 8E) or injected with Glucantime (Figure $8 \mathrm{G}$ ) presented few amastigotes, with fewer inflammatory cells than the control. A healing process could be observed evidenced by the presence of collagen fibers in the dermis of treated mice.

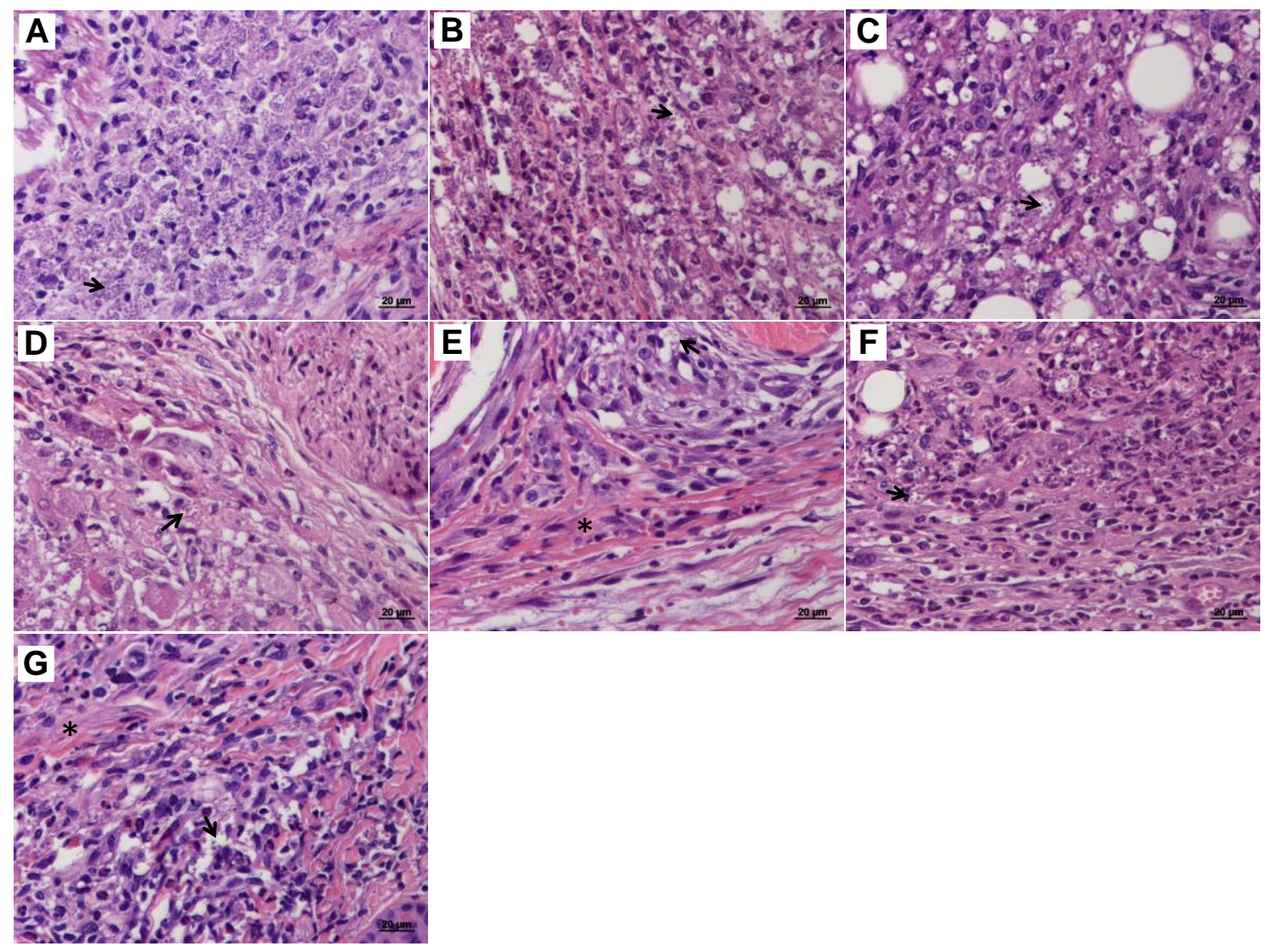

Figure 8. Histological images of excised BALB/c mouse skin of $L$. (L.) amazonensis infected mice; A: untreated, B: treated topically with blank SNEDDS gel, C: treated topically with blank SNEDDS, D: treated intralesionally (IL) with BPQ in DMSO, E: treated topically with BPQ-SNEDDS gel, F: treated topically with BPQ-SNEDDS, G: treated IL with Glucantime. Key: Black arrows indicate amastigotes and asterisk

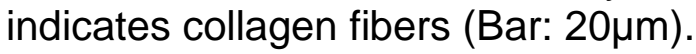




\subsection{Discussion}

Available topical therapies for $\mathrm{CL}$ are only indicated for the least severe forms of $\mathrm{CL}$ without the risk of dissemination and development of MCL, as topical therapies have shown variable efficacy especially when $\mathrm{CL}$ is caused by New World Leishmania species. Although drugs with good efficacy against VL species such as liposomal amphotericin B have recently shown promise in $\mathrm{CL}$ and $\mathrm{MCL}\left(3 \mathrm{mg} \mathrm{kg} \mathrm{kg}^{-1}\right.$ intravenously for $5-20$ days) [32, 33], topical therapies remain a preferred option due to cost, need for trained personnel for administration and systemic toxicity. Buparvaquone (BPQ) is a hydroxynapthoquinone stemming out from the open access Malaria Box with favourable physicochemical characteristics for skin penetration. BPQ failed to translate as a viable treatment for $V L$ due to its poor aqueous solubility and liver degradation [1]. Here, we demonstrate a readily scalable platform technology for eliciting topical nanomedicines from generally regarded as safe excipients for repurposed drugs such as BPQ.

We have shown topical efficacy of BPQ nano-enabled gels by monitoring both lesion size and progression as well as parasite burden by limiting-dilution assays (LDAs). The proposed nano-enabled hydrogel (1\%) significantly reduced parasite burden after 7 days of daily administration ( $\mathrm{p}<0.05,49$ days post-infection) and lesion size compared with the untreated control $(p<0.05,35$ days post-infection). Previous studies have indicated that the timing for start of treatment greatly influences treatment outcome for BPQ topical treatments. Previous studies have shown that a hydrous $\mathrm{BPQ}$ gel $(0.2 \%)$ and a water-in-oil emulsion (1\% cream) significantly reduced cutaneous Old World parasite burden (L. major) after 20 daily doses $(\mathrm{p}<0.05,22$ days post-infection) and lesion size, compared with the untreated control ( $p<0.0001,16$ days post-infection) [8]. Topical treatment was started 3,10 and 23 
days post-infection and when treatment started just 3 days post-infection, treatment was more effective in reducing lesion progression, when compared with treatment started 10 or 23 days post-infection [8]. Although topical efficacy is likely to be more easily demonstrated in the early stages of infection than on well-established lesions, the reverse is true for clinical environments, where patients usually only seek medical intervention on well-established lesions. In our study, animals were treated 28 days post-infection with New World species (L. (L.) amazonensis) and received treatment for only seven consecutive days. BALB/c mice develop chronic progressive lesions and parasites are known to disseminate from the infection site within hours [8], which is likely to be the case with more difficult to treat $L$. amazonensis infections. It is important to note that reduction in lesion size was in accordance to reduction in parasite load. LDAs are based on the ability of viable parasites to grow and replicate in culture. BPQ-SNEDDS gel application for 7 days has resulted in a near complete reduction of parasite load $(99.989 \pm 0.019 \%)$ that was not significantly different to intralesionally administered Glucantime (99.873 \pm $0.204 \%$ ) (Figure 7C). Reduction in parasitism was also shown histologically (Figure 8). Intralesional administration of $\mathrm{BPQ}$ in DMSO showed good ability to reduce parasite load (98.241 $\pm 0.491 \%)$ but not when solubilised in SNEDDS and topically applied $(79.814 \pm 5.852 \%)$. SNEDDS are isotropic mixtures known to spontaneously emulsify in aqueous environments to yield stable nanoemulsions. However, when water availability is limited, particle size of produced emulsion can be affected explaining observed efficacy. It is also important to note that topical BPQ-SNEDDS hydrogels were well tolerated in vivo (Figures 6 and 8) and ex vivo (Figure 5) and histological studies revealed limited inflammatory infiltrates and demonstrated healing (Figure 8). 
In vivo efficacy is supported by ex vivo permeability and in vivo tape stripping studies. BPQ-SNEDDS gels are loaded with high amounts of BPQ than previous reported hydrous gels [10] as BPQ-SNEDDS can solubilise high amounts of BPQ $\left(20 \mathrm{mg} \mathrm{g}^{-1}\right)$. Particle size of our nano-enabled BPQ formulations is consistently below $300 \mathrm{~nm}$ and aqueous dispersions showed adequate colloidal stability (> $-25 \mathrm{mV}$ ) (Table 1) with near spherical morphology with an electrodense core (Figure 1). Particles of similar shape and morphology are released from BPQ-SNEDDS gels (Figure 1). A linear flux was observed for BPQ-SNEDDS and their gels and with minimal lag time (Figure 2, Table 2). BPQ-SNEDDS and their hydrogels possess high flux across non-infected mouse skin ex vivo of $182.4 \pm 63.0 \mu \mathrm{g} \mathrm{cm}^{-2} \mathrm{~h}^{-1}$ and $57.6 \pm 10.8 \mu \mathrm{g} \mathrm{cm}^{-2} \mathrm{~h}^{-1}$ respectively localising BPQ within the skin in clinically effective concentrations $(227.0 \pm 45.9 \mu \mathrm{g}$ and $103.8 \pm 33.8 \mu \mathrm{g})$ respectively based on in vitro efficacy studies (L. amazonensis amastigotes $\mathrm{IC}_{50}: 773 \mathrm{ng} \mathrm{mL} \mathrm{L}^{-1}$, Table 5). Since BPQ is highly lipophilic, hydrogels would provide the greatest flux due to greater thermodynamic activity within this environment, and thus the higher the drug loading, the highest the thermodynamic activity and flux would be. This explains why the flux obtained is 123 -fold higher than previous reports for BPQ hydrous gels $(0.08$ $\% \mathrm{w} / \mathrm{w})\left(0.47 \pm 0.03 \mu \mathrm{g} \mathrm{cm}-2 \mathrm{~h}^{-1}\right)$ that contained 12.5-fold lower BPQ.

$\mathrm{BPQ}$ release from nano-emulsifying drug delivery systems incorporated into a crosslinked polyacrylate polymers (carbomers) fitted better zero-order kinetics indicating a steady rate of release over time independent of concentration. The hydrogel could act as reservoir from which BPQ-SNEDDS particles are released followed by BPQ release from the SNEDDS into the SC providing sustained delivery over time. BPQSNEDDS drug release was best described by the Korsmeyer-Peppas model. The release exponent $(n)$ for BPQ-SNEDDS model was above 1.4 (Table 3), which 
indicates a Super Case II drug transport mechanism $(n>1)$ involving emulsification and destabilisation upon dilution to release the drug.

Previous studies of $\mathrm{BPQ}$ topical formulations showed a different rank order of penetration between mouse and human skin [10] depending on the penetration enhancing strategy employed. However, this is not expected to be the case for our developed SNEDDS, which have shown similar rank order of penetration between mouse and human (eyelid) skin for anaesthetics [12], which indicates that our formulations optimised both in vitro and in vivo on animal skin are likely to behave similarly when applied on human skin.

$\mathrm{BPQ}$ was not able to permeate healthy skin in previous studies, but shown to be able to permeate $L$. major infected skin $\left(0.17 \pm 0.1 \mu \mathrm{g} \mathrm{cm}^{-2} \mathrm{~h}^{-1}\right.$, lag time: $\left.25.32 \pm 3.1 \mathrm{~h}\right)$ [11]. Our BPQ SNEDDS gel showed no determinable lag time and BPQ SNEDDS showed a short lag time $(0.454 \pm 0.191 \mathrm{~h})$ (Table 2$)$. The low lag time indicates that SNEDDS release from hydrogels is not hindered, possibly due to regions of low microviscosity of the carbomer gels as only $50 \%$ of the polymer chains are ionised at pH $6.0[28,34]$. Histology studies of L. major infected BALB/c skin revealed an upward movement of the skin layers due to the infiltration of cells, a disruption of the continuity of the epidermal and dermal skin layers at the centre of the lesion, epidermal hyperplasia, abundant presence of macrophage-like cells in the dermal and lower epidermal layers and reduced density of collagen fibres networks compared to uninfected skin [11]. Transepidermal water loss was increased 2-fold in infected mice when a nodule on the skin was visible and thus prior treatment is initiated [11]. The latter is significant as utilising a hydrogel will allow the restoration of the skin hydration and reduce damage to the skin barrier. 
In vivo tape stripping was undertaken to access whether $\mathrm{BPQ}$ levels within the SC and dermis after $0.5,1,2$, and 4 hours of exposure to the nano-enabled formulations would result in therapeutic levels. Complete removal of the SC was possible under our experimental conditions (histology data not shown). BPQ-SNEDDS gels were able to localise therapeutic levels of BPQ in the SC and dermis without resulting in determinable levels of BPQ in the homogenised skin at any time point. The use of animals that were not nude can explain the higher deviation in BPQ levels observed with gels as could limit the contact with the gels compared to liquid SNEDDS (Figure 3C, 3D, 4, Table 4). BPQ-SNEDDS were able to elicit high levels of BPQ in the SC and dermis and permeation remained constant with steady state being reached after an hour of exposure. Transcutol P, although used within approved FDA levels for skin products, can cause swelling of the lipid bilayer structures resulting in an intracutaneous drug depot effect, which improves drug retention within the skin and allows drugs to be released in a sustained manner [12]. Significant levels of BPQ were also quantified in the tape stripped homogenised skin indicating that potentially some BPQ might be reaching the bloodstream. The application of metallic microneedles increased BPQ permeation across the skin from BPQ-SNEDDS and resulted in higher levels in the stratum corneum, dermis (1.46-fold after 1 hour) and homogenised skin compared to SNEDDS alone (Table 4, Figure 3E, 3F, 4) $(p<0.05$, Student T-test). After pores were healed (after $1 \mathrm{~h}$ ) permeation remained high over time. Initial microneedle application limits available surface area for diffusion that is restored after healing has taken place. Intracellular permeation across scar tissue from SNEDDS might be higher but further studies are needed to confirm this. As we have not used microneedles in our in vivo efficacy studies, we cannot conclude whether this could be utilised to allow drug permeation across Leishmania infected 
skin lesions. Our pharmacokinetic studies can only provide information regarding BPQ uptake across mouse skin, but as designed cannot be utilised to calculate elimination constants or other pharmacokinetic parameters, which would require ideally larger species.

\subsection{Conclusions}

In vivo pharmacokinetic and efficacy results complimented with histology studies highlight the potential for clinical translation of the proposed nano-enabled buparvaquone hydrogels as a non-invasive topical treatment for neglected tropical diseases such as CL even when caused by New World Leishmania species. The developed platform technology is readily scalable, cost-effective and prepared by generally regarded as safe excipients can enable the delivery of skin impermeable APIs

\section{Acknowledgements}

The authors thank Prof Simon Cragg (University of Portsmouth) for sharing his expertise and helping with TEM imaging.

\section{Funding}

This work was funded by the Royal Society (RG130542), the Fundação de Amparo à Pesquisa do Estado de São Paulo (FAPESP: 2016/00468-0) and an Ibero-American Universities Union Research Collaboration Fund (Unión Iberoamericana de Universidades; ENF03-2017) that allowed student mobility exchange between UCM 
(Madrid, Spain) and USP (Sao Paulo, Brazil). Larry Statts was supported by an

Erasmus $^{+}$to undertake research placements at UCM, Madrid, Spain.

\section{References}

1. Smith, L., et al., Orally Bioavailable and Effective Buparvaquone Lipid-Based Nanomedicines for Visceral Leishmaniasis. Mol Pharm, 2018. 15(7): p. 2570-2583.

2. Alvar, J., et al., Leishmaniasis worldwide and global estimates of its incidence. PLoS One, 2012. 7(5): p. e35671.

3. Moreno, E., et al., Nanoparticles as multifunctional devices for the topical treatment of cutaneous leishmaniasis. Expert Opin Drug Deliv, 2014. 11(4): p. 579-97.

4. Uranw, S., et al., The household costs of visceral leishmaniasis care in south-eastern Nepal. PLoS Negl Trop Dis, 2013. 7(2): p. e2062.

5. Serrano Lopez, D.R. and A. Lalatsa, Oral amphotericin B: The journey from bench to market. Journal of Drug Delivery Science and Technology, 2017. 42: p. 75-85.

6. Passero, L.F.D., et al., Conventional Versus Natural Alternative Treatments for Leishmaniasis: A Review. Curr Top Med Chem, 2018. 18(15): p. 1275-1286.

7. Fry, M. and M. Pudney, Site of action of the antimalarial hydroxynaphthoquinone, 2[trans-4-(4'-chlorophenyl) cyclohexyl]-3-hydroxy-1,4-naphthoquinone (566C80). Biochem Pharmacol, 1992. 43(7): p. 1545-53.

8. Soni, M.P., et al., Buparvaquone loaded solid lipid nanoparticles for targeted delivery in theleriosis. J Pharm Bioallied Sci, 2014. 6(1): p. 22-30.

9. Garnier, T., et al., In vivo studies on the antileishmanial activity of buparvaquone and its prodrugs. J Antimicrob Chemother, 2007. 60(4): p. 802-10.

10. Garnier, T., et al., Topical buparvaquone formulations for the treatment of cutaneous leishmaniasis. J Pharm Pharmacol, 2007. 59(1): p. 41-9.

11. Van Bocxlaer, K., et al., Drug permeation and barrier damage in Leishmania-infected mouse skin. J Antimicrob Chemother, 2016. 71(6): p. 1578-85.

12. Lalatsa, A., et al., Transcutaneous Anaesthetic Nano-enabled Hydrogels for Eyelid Surgery. International Journal of Pharmaceutics, 2019. 577: p. 119003.

13. Lalatsa, A., et al., Developing transcutaneous nanoenabled anaesthetics for eyelid surgery. Br J Ophthalmol, 2016. 100(6): p. 871-6.

14. Lalatsa, A., et al., Delivery of peptides to the blood and brain after oral uptake of quaternary ammonium palmitoyl glycol chitosan nanoparticles. Mol Pharm, 2012. 9(6): p. 1764-74.

15. Lalatsa, A., et al., Chitosan amphiphile coating of peptide nanofibres reduces liver uptake and delivers the peptide to the brain on intravenous administration. J Control Release, 2015. 197: p. 87-96.

16. Dea-Ayuela, M.A., et al., In vivo and in vitro anti-leishmanial activities of 4-nitro-Npyrimidin- and N-pyrazin-2-ylbenzenesulfonamides, and N2-(4-nitrophenyl)-N1propylglycinamide. Bioorg Med Chem, 2009. 17(21): p. 7449-56.

17. Bilbao-Ramos, P., et al., A fluorometric method for evaluation of pharmacological activity against intracellular Leishmania amastigotes. J Microbiol Methods, 2012. 89(1): p. 8-11.

18. Rolon, M., et al., Development of resazurin microtiter assay for drug sensibility testing of Trypanosoma cruzi epimastigotes. Parasitol Res, 2006. 99(2): p. 103-7.

19. Serrano, D.R., et al., Oral particle uptake and organ targeting drives the activity of amphotericin B nanoparticles. Mol Pharm, 2015. 12(2): p. 420-31. 
20. Venkatesh, G., et al., In vitro and in vivo evaluation of self-microemulsifying drug delivery system of buparvaquone. Drug Dev Ind Pharm, 2010. 36(6): p. 735-45.

21. Phillips, C.A. and B.B. Michniak, Transdermal delivery of drugs with differing lipophilicities using azone analogs as dermal penetration enhancers. J Pharm Sci, 1995. 84(12): p. 1427-33.

22. Ganem-Quintanar, A., et al., Design and evaluation of a self-adhesive naproxenloaded film prepared from a nanoparticle dispersion. J Nanosci Nanotechnol, 2006. 6(9-10): p. 3235-41.

23. Dickel, H., et al., Standardized tape stripping: a practical and reproducible protocol to uniformly reduce the stratum corneum. Skin Pharmacol Physiol, 2010. 23(5): p. 259-65.

24. Serrano, D.R., et al., Tuning the Transdermal Delivery of Hydroquinone upon Formulation with Novel Permeation Enhancers. Pharmaceutics, 2019. 11(4).

25. Yamamoto, E.S., et al., The Effect of Ursolic Acid on Leishmania (Leishmania) amazonensis Is Related to Programed Cell Death and Presents Therapeutic Potential in Experimental Cutaneous Leishmaniasis. PLoS One, 2015. 10(12): p. e0144946.

26. Trinconi, C.T., et al., Efficacy of tamoxifen and miltefosine combined therapy for cutaneous leishmaniasis in the murine model of infection with Leishmania amazonensis. J Antimicrob Chemother, 2016. 71(5): p. 1314-22.

27. Carvalho, A.K., et al., Differential Recruitment of Dendritic Cells Subsets to Lymph Nodes Correlates with a Protective or Permissive T-Cell Response during Leishmania (Viannia) Braziliensis or Leishmania (Leishmania) Amazonensis Infection. Mediators Inflamm, 2016. 2016: p. 7068287.

28. Chakraborti, C.K., S. Sahoo, and P.K. Behera, Role of different biodegradable polymers on the permeability of ciprofloxacin. J Adv Pharm Technol Res, 2014. 5(3): p. 140-6.

29. Kietzmann, M., D. Lubach, and H.J. Heeren, The mouse epidermis as a model in skin pharmacology: influence of age and sex on epidermal metabolic reactions and their circadian rhythms. Lab Anim, 1990. 24(4): p. 321-7.

30. Reddy, M.B., et al., Determining dermal absorption parameters in vivo from tape strip data. Pharm Res, 2002. 19(3): p. 292-8.

31. Ioset, J.-R., et al., Drug Screening for Kinetoplastids Diseases: A Training Manual for Screening in Neglected Diseases, D.a.P.-A.S. Network, Editor. 2009.

32. Sundar, S. and J. Chakravarty, An update on pharmacotherapy for leishmaniasis. Expert Opin Pharmacother, 2015. 16(2): p. 237-52.

33. Wijnant, G.J., et al., Relation between Skin Pharmacokinetics and Efficacy in AmBisome Treatment of Murine Cutaneous Leishmaniasis. Antimicrob Agents Chemother, 2018. 62(3).

34. Majid Khan, G. and J.B. Zhu, Ibuprofen release kinetics from controlled-release tablets granulated with aqueous polymeric dispersion of ethylcellulose II: influence of several parameters and coexcipients. J Control Release, 1998. 56(1-3): p. 127-34. 
Figure Legends:

Figure 1. TEM images of aqueous dispersions of BPQ-SNEDDS (A) and BPQSNEDDS gel (B) (1 in $2000 \mathrm{w} / \mathrm{w})$ stained with 1\% uranyl acetate. Bars: 50 and 100 $\mathrm{nm}$ respectively.

Figure 2. Flux of BPQ across artificial skin/STRAT-M membraned (A) and BALB/C excised mouse skin (B) [BPQ-SNEDDS (2\%, black squares), BPQ-SNEDDS gels $(1 \%$, red cycles $)]$.

Figure 3. In vivo tape stripping after exposure to nano-enabled formulations; Amount of $B P Q$ per tape $(1 \mathrm{~A})$ and cumulative $B P Q$ amount (1B) after exposure to $B P Q-$ SNEDDS over $0.5 \mathrm{~h}$ (black squares), $1 \mathrm{~h}$ (red cycles), $2 \mathrm{~h}$ (green triangles) and $4 \mathrm{~h}$ (blue inverted triangles). Amount of BPQ per tape (1C) and cumulative BPQ amount (1D) after exposure to BPQ-SNEDDS gels over $0.5 \mathrm{~h}$ (black squares), $1 \mathrm{~h}$ (red cycles), $2 \mathrm{~h}$ (green triangles) and $4 \mathrm{~h}$ (blue inverted triangles). Amount of BPQ per tape $(1 \mathrm{~A})$ and cumulative $\mathrm{BPQ}$ amount $(1 \mathrm{~B})$ after exposure to metallic microneedles $(0.4 \mathrm{~mm}, 1 \mathrm{sec}$ ) and BPQ-SNEDDS over $0.5 \mathrm{~h}$ (black squares), $1 \mathrm{~h}$ (red cycles), $2 \mathrm{~h}$ (green triangles) and $4 \mathrm{~h}$ (blue inverted triangles).

Figure 4. Amount of $B P Q$ recovered in $S C(A)$, dermis $(B)$ and homogenised skin (C) over time after exposure to BPQ-SNEDDS (black circles), BPQ-SNEDDS gels (red squares), and metallic microneedles $(0.4 \mathrm{~mm}, 1 \mathrm{sec}$ ) and BPQ-SNEDDS (blue triangles) $(n=3)$. SD stands for standard deviation.

Figure 5. Micrographs of excised BALB/c mouse skin; Untreated skin (A: $x 4$ magnification and B: $x 10$ magnification), skin exposed to BPQ-SNEDDS for 6 hours (C: $x 4$ magnification and D: $x 10$ magnification), and skin exposed to BPQ-SNEDDS gels for 6 hours (E: $x 4$ magnification and $F$ : $x 10$ magnification).

Figure 6. Micrographs of excised BALB/c mouse skin after in vivo administration of topical formulations; BALB/C skin exposed to BPQ-SNEDDS for 2 hours (A: $x 4$ magnification) and BPQ-SNEDDS gels for 2 hours (B: $x 4$ magnification), and BALB/C skin perforated by microneedles and exposed to BPQ-SNEDDS for 2 hours (C,D,E,F: $x 4$ magnification). Healing of microneedle punctures is visible.

Figure 7. In vivo efficacy of nanoenabled gels in $L$. (L.) amazonensis infected BALB/C mice ( $n=8 /$ group). Macroscopic images of lesions of infected untreated and treated animals (A), lesion size (B) and parasite load (C) is shown for mice that were subcutaneously infected into the base of the tail with $10^{6}$ promastigotes of $L$. (L.) amazonensis. Four weeks after infection, mice were divided randomly into groups and treated once daily for 7 days; infected control (no treatment, black line), BPQ in DMSO intralesionally (IL) $\left(50 \mu \mathrm{L}, 45 \mathrm{mg} \mathrm{kg}^{-1}\right.$, blue line), BPQ-SNEDDS topically (0.05g, $45 \mathrm{mg} \mathrm{kg}^{-1}$, red line), BPQ-SNEDDS gels topically $\left(0.15 \mathrm{~g}, 45 \mathrm{mg} \mathrm{kg}^{-1}\right.$, purple 
line), Glucantime IL(25 $\mu \mathrm{L}, 100 \mathrm{mg} \mathrm{kg}^{-1}$, green line), blank SNEDDS topically $(0.05 \mathrm{~g}$, turquoise line), blank SNEDDS gels topically (0.15 g, yellow line). No statistical differences in lesion size were obtained for blank SNEDDS, blank-SNEDDS gels and BPQ in DMSO at any time point (Kruskal Wallis test, 95\% level of significance). At week 5,6 , and 7, there were statistical differences (reduction in lesion size) between the untreated group and groups that received Glucantime (week $5 \mathrm{p}$ value:0.0282, week 6 p value: $<0.0001$, week 7 p value: $<0.0001)$, BPQ-SNEDDS gels topically (week 5 p value: $<0.0001$, week 6 p value: 0.0002 , week $7 p$ value: $<0.0001$ ) and BPQ-SNEDDS topically (week $5 p$ value:0.0002, week $6 p$ value: 0.0099 , week $7 p$ value: 0.0031) (Kruskal Wallis test, 95\% level of significance). BPQ in DMSO (IL), BPQ-SNEDDS topically, BPQ-SNEDDS gel topically, Glucantime (IL) have a significantly different parasite load compared to the untreated group $(p<0.0001$, OneWay Anova, Dunnett's test). Analysis was undertaken using Graphpad Prism 8.02 and results are shown as mean \pm SD.

Figure 8. Histological images of excised BALB/c mouse skin of L. (L.) amazonensis infected mice; A: untreated, B: treated topically with blank SNEDDS gel, C: treated topically with blank SNEDDS, D: treated intralesionally (IL) with BPQ in DMSO, E: treated topically with BPQ-SNEDDS gel, F: treated topically with BPQ-SNEDDS, G: treated IL with Glucantime. Key: Black arrows indicate amastigotes and asterisk indicates collagen fibers (Bar: $20 \mu \mathrm{m}$ ). 